\title{
Dense Crystalline Dimer Packings of Regular Tetrahedra
}

\author{
Elizabeth R. Chen • Michael Engel • \\ Sharon C. Glotzer
}

Received: 27 December 2009 / Revised: 20 April 2010 / Accepted: 16 May 2010 /

Published online: 17 July 2010

(C) Springer Science+Business Media, LLC 2010

\begin{abstract}
We present the densest known packing of regular tetrahedra with density $\phi=\frac{4000}{4671}=0.856347 \ldots$ Like the recently discovered packings of Kallus et al. and Torquato-Jiao, our packing is crystalline with a unit cell of four tetrahedra forming two triangular dipyramids (dimer clusters). We show that our packing has maximal density within a three-parameter family of dimer packings. Numerical compressions starting from random configurations suggest that the packing may be optimal at least for small cells with up to 16 tetrahedra and periodic boundaries.
\end{abstract}

Keywords Crystallography $\cdot$ Packing $\cdot$ Regular solid $\cdot$ Hilbert problem

\section{Introduction}

The problem of the packing of tetrahedra, which has modern-day applications to such wide-ranging topics as metamaterials with novel optical properties, nanomaterials [1-3], and virus formation, hails back to the early Greeks [4]. Aristotle, in discussing the assignment of geometrical figures to "heavenly bodies", mistakenly believed that regular tetrahedra ("pyramids") tile (Euclidean) space perfectly. Nearly 1800 years

\footnotetext{
E.R. Chen

Department of Mathematics, University of Michigan, Ann Arbor, MI 48109, USA

e-mail: bethchen@umich.edu

M. Engel $(\bowtie) \cdot$ S.C. Glotzer

Department of Chemical Engineering, University of Michigan, Ann Arbor, MI 48109, USA

e-mail: engelmm@umich.edu

S.C. Glotzer

Department of Materials Science and Engineering, University of Michigan, Ann Arbor, MI 48109, USA

e-mail: sglotzer@umich.edu
} 
later, Johannes Müller (a.k.a. Regiomontanus, 1436-1476) contradicted Aristotle's claim [5, 6]. 400 years after Müller, Minkowski showed that the densest lattice packing of any convex body must satisfy certain constraints [7] and mistakenly argued that the densest lattice packing of tetrahedra (i.e. one tetrahedron per unit cell) had density $\phi=\frac{9}{38}$ [8]. In 1900, Hilbert posed the problem of the densest lattice packing of regular tetrahedra as a special case of the 18th of his famous list of problems [9]. Grömer conjectured [10], and Hoylman later proved [11], that the densest lattice packing of a single tetrahedron is $\phi=\frac{18}{49}$.

In 1972, Stanislaw Ulam conjectured that spheres would have the lowest maximum packing density of all convex bodies, including tetrahedra [12]. That density is $\phi_{\text {sphere }}=\frac{\pi}{\sqrt{18}}=0.740480 \ldots$ [13]. In 2000, Betke and Henk developed an efficient computer algorithm to compute the densest lattice packing of any convex body, and applied it to the Archimedean solids [14]. Conway and Torquato [15] used Betke and Henk's algorithm to compute the packing density of tetrahedra derived from the densest lattice packing of icosahedra, and also examined other promising packings. All of them packed worse than spheres, with a maximum packing fraction $\phi \approx 0.7175$. This led them to suggest the tantalizing possibility that the Ulam conjecture might, in fact, fail for tetrahedra. They further proposed that tetrahedra might have the lowest packing density of all convex bodies. Motivated by this, Chaikin and coworkers [16] performed experiments on tetrahedral dice packed in spherical and cylindrical containers. They reported random packings with densities as high as $0.75 \pm 0.03$ [16] and $0.76 \pm 0.02$ [17], with the error arising from the rounded dice vertices and edges [17].

In 2008, Chen proposed a packing of nonamers (nine tetrahedra forming two pentagonal dipyramids that share one tetrahedron) arranged in layers, whose density $(\phi \approx 0.7786)$ clearly exceeded, for the first time, the maximum packing density of spheres [18]. Since Chen's publication, a flurry of activity has resulted in successively higher and higher packing densities for tetrahedra. It was soon demonstrated that the nonamer crystal could be numerically compressed to slightly higher packing densities ( $\phi=0.7837$ [19] and $\phi=0.7820$ [20]). The first disordered (i.e. non-periodic) packing of tetrahedra to exceed the maximum packing density of spheres was reported by Haji-Akbari et al. [19, 21]; they obtained a packing density of $\phi=0.7858$ using Monte Carlo simulation of systems of 8000 tetrahedra compressed from random initial conditions. Their disordered packing contains a preponderance of pentagonal dipyramids, arranged randomly and thus differently than in the Chen structure, along with other motifs such as icosahedra.

Previously, all dense ordered packings proposed for tetrahedra were based on analytical construction (or numerical compressions thereof). Haji-Akbari et al. showed, using MC simulations of initially random systems containing up to nearly 22,000 tetrahedra, that at packing densities $\phi>0.5$ an equilibrium fluid of hard tetrahedra spontaneously transforms to a dodecagonal quasicrystal, which can be compressed to $\phi=0.8324$ [21]. By numerically constructing and then compressing four unit cells of a periodic quasicrystal approximant with an 82-tetrahedron unit cell, they obtained a packing density as high as $\phi=0.8503$.

Motivated by a numerical search, Kallus, Elser and Gravel [22] found a oneparameter family of dimer cluster packings obtained via an analytical construction with a density of $\phi=\frac{100}{117}=0.854700 \ldots$, exceeding the density of the quasicrystal approximant. Each dimer cluster contains two face-sharing tetrahedra, and is 
equivalent to a triangular dipyramid (or bipyramid). Two dimers comprise a single unit cell. Torquato and Jiao generalized the analytical construction of Kallus et al. to a two-parameter family of packings, and obtained an even denser packing with $\phi=\frac{12250}{14319}=0.855506 \ldots[23]$.

In this paper, we further generalize the Kallus et al. and Torquato-Jiao family of dimer cluster packings to consider a three-parameter family of packings. We obtain a maximum packing density of $\phi=\frac{4000}{4671}=0.856347 \ldots$ as the optimal solution within the three-parameter family and show that the Kallus et al. and Torquato-Jiao packings are special cases of our more general construction. Furthermore, we present numerical compression simulations of small systems of tetrahedra using standard isobaric Monte Carlo with variable simulation cell shape. For systems with 4, 8, 12 and 16 tetrahedra with initially random configurations, the analytically predicted dimer crystal with $\phi=0.856347$ is recovered.

\section{Analytical Construction}

\subsection{Double Dimer Configurations}

Definition 1 A dimer (cluster) is a basic building block formed by two face-sharing regular tetrahedra arranged into a triangular dipyramid. The vertices of a positive dimer are chosen up to translation as

$$
\begin{aligned}
o & =\langle+2,+2,+2\rangle, \\
p=\langle+2,-1,-1\rangle, \quad q & =\langle-1,+2,-1\rangle, \quad r=\langle-1,-1,+2\rangle, \\
s & =\langle-2,-2,-2\rangle,
\end{aligned}
$$

such that $p, q, r$ span the common face. A negative dimer is related to a positive dimer by inversion. The positive dimer with vertices $o, p, q, r, s$, centered at the origin, is called $+\mathbf{F}_{2}$; the corresponding negative dimer with vertices $-o,-p,-q$, $-r,-s$, centered at the origin, is called $-\mathbf{F}_{2}$.

Definition 2 A crystalline arrangement of tetrahedra with four tetrahedra in the unit cell forming one negative dimer and one positive dimer is called a double dimer configuration. If the configuration is free of overlaps, then it is a double dimer packing.

The set of possible double dimer configurations $\mathcal{P}$ is a set of translates of the two dimers. We place the positive dimer $+\mathbf{F}_{2}$ at the origin and translate it by the lattice $\mathbf{L}^{+}$. The negative dimer $-\mathbf{F}_{2}$ is placed by the lattice coset $\mathbf{L}^{-}$. Without loss of generality we can assume that $\mathbf{L}^{+}$is spanned by $a+b, b+c, c+a$ and choose the offset of $\mathbf{L}^{-}$as $d+a$ (equivalently $d+b$, or $\left.d+c\right)$, where the lattice vectors $(a, b$, $c$ ) and the offset vector $d$ are free to vary. This means that

$$
\begin{aligned}
& \mathbf{L}^{+}=\left\{n_{a} a+n_{b} b+n_{c} c \mid n_{a}+n_{b}+n_{c}=0 \bmod 2\right\}, \\
& \mathbf{L}^{-}=\left\{n_{a} a+n_{b} b+n_{c} c \mid n_{a}+n_{b}+n_{c}=1 \bmod 2\right\}+d .
\end{aligned}
$$


We say a dimer is part of the $n$-th layer with $n=n_{a}+n_{b}+n_{c}$. The even (odd) layers contain positive (negative) dimers.

The lattice and offset vectors contain 12 coordinate variables, which means the set $\mathcal{P}$ is a 12-dimensional linear space. However, many of the configurations have overlaps, and thus are not packings.

\subsection{Three-Parameter Family}

In order to verify that the dimers of a double dimer configuration are not overlapping (i.e. that it is a packing), intersections of dimers with their neighbors are studied. It is sufficient to consider the dimer at the origin:

Lemma 1 The space group of a double dimer configuration acts transitively on the dimers.

Proof The inversion $x \mapsto d+a-x$ maps positive dimers to negative dimers.

Intersections can occur between three types of simplices forming the surface of a dimer: triangular faces, edges, and vertices. Given two simplices, we say the incidence condition for these simplices holds if their intersection is non-empty. Incidence conditions are of either face-to-face, face-to-edge, face-to-vertex, edge-to-edge, edgeto-vertex or vertex-to-vertex type.

Next, we replace the simplices by affine subspaces and use the notation $V[p]=$ $\{p\}$ to be the vertex $p, E[p, q]=\{p+\kappa(q-p): \kappa \in \mathbb{R}\}$ to be the line containing the edge from $p$ to $q$, and $F[p, q, r]=\{p+\kappa(q-p)+\lambda(r-p): \kappa, \lambda \in \mathbb{R}\}$ to be the plane containing the triangular face spanned by $p, q$, and $r$. We say the linear incidence condition holds if the intersection of two affine subspaces is non-empty. In the following we will consider linear incidence conditions only. They are easier to handle analytically and can, in certain cases, be used as sufficient conditions for the absence of overlaps.

Lemma 2 Given the nine linear incidence conditions

$$
\begin{array}{ll}
\mathbf{G}_{a}^{+}: & F[o, p, q] \cap(d-F[o, p, q]+a) \neq \emptyset, \\
\mathbf{G}_{b}^{+}: & F[o, q, r] \cap(d-F[o, q, r]+b) \neq \emptyset, \\
\mathbf{G}_{c}^{+}: & F[o, r, p] \cap(d-F[o, r, p]+c) \neq \emptyset, \\
\mathbf{G}_{a+b+c}^{+}: & F[o, r, p] \cap(d-F[o, r, p]+a+b+c) \neq \emptyset, \\
\mathbf{G}_{a}^{-}: & F[s, r, q] \cap(d-F[s, r, q]-a) \neq \emptyset, \\
\mathbf{G}_{b}^{-}: & F[s, p, r] \cap(d-F[s, p, r]-b) \neq \emptyset, \\
\mathbf{G}_{c}^{-}: & F[s, q, p] \cap(d-F[s, q, p]-c) \neq \emptyset, \\
\mathbf{G}_{a+b+c}^{-}: & F[s, q, p] \cap(d-F[s, q, p]-a-b-c) \neq \emptyset, \\
\mathbf{G}_{a+b}: & E[o, q] \cap(E[s, r]+a+b) \neq \emptyset,
\end{array}
$$


the collection $\mathcal{P}^{\prime}=\left\{p \in \mathcal{P}\right.$ : the conditions $\mathbf{G}_{a}^{ \pm}, \mathbf{G}_{b}^{ \pm}, \mathbf{G}_{c}^{ \pm}, \mathbf{G}_{a+b+c}^{ \pm}, \mathbf{G}_{a+b}$ hold $\}$ is a three-dimensional linear space.

Proof The nine linear incidence conditions are sufficient for the statement that the dimer at the origin is not overlapping with the neighbors at $d \pm a, d \pm b, d \pm c$, $d \pm(a+b+c)$, and $\pm(a+b)$. Note that $\mathbf{L}^{+}$is invariant under inversion at the origin, so that we do not have to consider the neighbors at $+(a+b)$ and $-(a+b)$ separately.

Since the planes involved in the face-to-face condition $\mathbf{G}_{a}^{+}$are parallel, they coincide if they are incident, so we can alternatively write $\mathbf{G}_{a}^{+}$as an equation:

$$
\mathbf{G}_{a}^{+}: \quad 2 o+\kappa(p-o)+\lambda(q-o)=d+a
$$

with parameters $\kappa, \lambda \in \mathbb{R}$. This equation is a vector equation and thus there is one scalar equation for each of its three components. Two of the scalar equations are used to eliminate $\kappa$ and $\lambda$. The remaining scalar equation is a single linear constraint for the components of the vectors $d$ and $a$. Similar constraints are obtained for the seven other face-to-face conditions. The edge-to-edge condition $\mathbf{G}_{a+b}$ corresponds to the equation

$$
\mathbf{G}_{a+b}: \quad o+\mu(q-o)=s+v(r-s)+a+b
$$

with parameters $\mu, v \in \mathbb{R}$. Again, a single linear constraint for the components of the vectors $a$ and $b$ is obtained. The system of linear constraints turns out to have rank 9 , so the dimension of the space of double dimer configurations $\mathcal{P}$ is reduced from 12 to 3. With the new scalar parameters $u, v, w$, the lattice vectors and the offset vector can be written as

$$
\begin{aligned}
& a=\left\langle\frac{27}{10}+u, \frac{21}{20}-v,-\frac{3}{20}+2 u+v\right\rangle, \\
& b=\left\langle-\frac{3}{10}-u, \frac{51}{20}+v, \frac{27}{20}-2 u-v\right\rangle, \\
& c=\left\langle\frac{129}{160}-u+2 v+2 w,-\frac{237}{320}+\frac{1}{2} u-v+3 w, \frac{753}{320}+\frac{1}{2} u-v+w\right\rangle, \\
& d=\left\langle\frac{1}{10}+u,-\frac{1}{20}+u+v,-\frac{1}{20}+u-v\right\rangle .
\end{aligned}
$$

Remark 1 (i) The eight face-to-face incidence conditions are important for dense packings, because the corresponding faces are parallel and therefore can be matched perfectly.

(ii) The face-to-face constraints imposed by $\mathbf{G}_{a+b+c}^{+}$and $\mathbf{G}_{a+b+c}^{-}$involve the faces spanned by $o, r, p$ and $s, q, p$, respectively. We balance the constraints with an opposing edge-to-edge constraint with the neighbors at $\pm(a+b)$ on the 'opposite' edge spanned by $o, q$ and $s, r$ in the form of the incidence condition $\mathbf{G}_{a+b}$. 


\subsection{Restricted Parameter Space}

We now specify a subset $\mathcal{P}^{\prime \prime} \subset \mathcal{P}^{\prime}$ of the three-parameter space that consists of double dimer packings only, and we later optimize density over this subset.

\section{Lemma 3 Consider the restricted parameter space}

$$
\mathcal{P}^{\prime \prime}=\left\{\langle u, v, w\rangle \in \mathcal{P}^{\prime}:\left|\frac{1}{2} u+2 v\right| \leq \frac{33}{320}+w \text { and }|v| \leq \frac{3}{64}-w\right\},
$$

where $\langle u, v, w\rangle$ is the parameterization in (6). Each double dimer configuration $p \in \mathcal{P}^{\prime \prime}$ is a packing.

Proof Since double dimer packings are transitive, it is sufficient to look for overlaps of $+\mathbf{F}_{2}$ only. We write a positive dimer as $+\mathbf{F}_{2}+n_{a} a+n_{b} b+n_{c} c$ and a negative dimer as $d-\mathbf{F}_{2}+n_{a} a+n_{b} b+n_{c} c$. The first part of the proof shows that we only need to consider the 26 neighbors with $-1 \leq n_{a}, n_{b}, n_{c} \leq 1$ when looking for overlaps. Note that $\mathcal{P}^{\prime \prime}$ has the shape of a sheared tetrahedron with the four vertices $e_{1,2}=\langle \pm 3 / 10,0,+3 / 64\rangle$ and $e_{3,4}=\langle\mp 3 / 5, \pm 3 / 20,-33 / 320\rangle$. We verify that for each configuration corresponding to one of the four extremal points $e_{j}$, the plane $a+\mathbb{R} b+\mathbb{R} c$ separates $+\mathbf{F}_{2}$ from $+\mathbf{F}_{2}+2 a$ and the plane $d / 2+a+\mathbb{R} b+\mathbb{R} c$ separates $+\mathbf{F}_{2}$ from $d-\mathbf{F}_{2}+2 a$. This shows that $n_{a} \leq 1$. By similar arguments we get $n_{a} \geq-1$ and $-1 \leq n_{b}, n_{c} \leq 1$. Since $a, b, c$ are linear in $u, v, w$ and $\mathcal{P}^{\prime \prime}$ is convex, the separating planes and the restrictions $-1 \leq n_{a}, n_{b}, n_{c} \leq 1$ remain valid for every double dimer configuration in $\mathcal{P}^{\prime \prime}$.

By construction of $\mathcal{P}^{\prime}$ in Lemma 2 we know that the ten dimers $d-\mathbf{F}_{2} \pm a$, $d-\mathbf{F}_{2} \pm b, d-\mathbf{F}_{2} \pm c, d-\mathbf{F}_{2} \pm(a+b+c),+\mathbf{F}_{2} \pm(a+b)$ do not overlap with $+\mathbf{F}_{2}$. Next, we find that the dimers $+\mathbf{F}_{2}$ and $d-\mathbf{F}_{2}+a+b-c$ are separated by the plane $F[o, p, q]$ for any configuration in $\mathcal{P}^{\prime \prime}$. We find similar planes for $d-\mathbf{F}_{2}+a-b+c$, $d-\mathbf{F}_{2}-a+b+c, d-\mathbf{F}_{2}-a-b+c, d-\mathbf{F}_{2}-a+b-c$, and $d-\mathbf{F}_{2}+a-b-c$, which shows that these six dimers cannot overlap with $+\mathbf{F}_{2}$. Finally note that pairs of positive neighbors are related by inversion symmetry. These considerations reduce the number of neighbors that have to be checked from 26 to five. The remaining ones are: $+\mathbf{F}_{2}+b+c,+\mathbf{F}_{2}+c+a,+\mathbf{F}_{2}+a-b,+\mathbf{F}_{2}+b-c,+\mathbf{F}_{2}+c-a$.

Consider the linear edge-to-edge and vertex-to-vertex incidence conditions

$$
\begin{array}{ll}
\mathbf{H}_{b+c}: & E[o, r] \cap(E[s, p]+b+c) \neq \emptyset, \\
\mathbf{H}_{c+a}: & E[o, p] \cap(E[s, q]+c+a) \neq \emptyset, \\
\mathbf{H}_{a-b}: & V[p] \cap(E[r, q]+a-b) \neq \emptyset, \\
\mathbf{H}_{b-c}: & V[q] \cap(F[s, p, r]+b-c) \neq \emptyset, \\
\mathbf{H}_{c-a}: & V[r] \cap(F[o, q, p]+c-a) \neq \emptyset .
\end{array}
$$

Similar to the linear incidence conditions in Lemma 2, the conditions $\mathbf{H}_{b+c}, \mathbf{H}_{c+a}$, $\mathbf{H}_{b-c}, \mathbf{H}_{c-a}$ can be written as four linear constraints for the parameters $u, v, w$, which correspond to four boundary planes. Each of the planes separates $\mathcal{P}^{\prime}$ into two halfspaces. Overlaps can only appear in one of the half-spaces, and the half-spaces that 
are free from overlaps are:

$$
\begin{array}{ll}
\mathbf{H}_{b+c}: & +\frac{1}{2} u+2 v-w \leq \frac{33}{320}, \\
\mathbf{H}_{c+a}: & -\frac{1}{2} u-2 v-w \leq \frac{33}{320}, \\
\mathbf{H}_{b-c}: & -v+w \leq \frac{3}{64}, \\
\mathbf{H}_{c-a}: & +v+w \leq \frac{3}{64} .
\end{array}
$$

The intersection of the four half-spaces is the restricted parameter space $\mathcal{P}^{\prime \prime}$.

The condition $\mathbf{H}_{a-b}$ is different from the other ones, since it is a vertex-to-edge condition. It is true if and only if both vertex-to-face conditions $V[p] \cap(F[o, r, q]+$ $a-b) \neq \emptyset$ and $V[p] \cap(F[s, r, q]+a-b) \neq \emptyset$ are true. We calculate the region of parameter space that is free from overlap using the half-spaces for these two conditions:

$$
\mathbf{H}_{a-b}: \quad-u \leq 0 \text { and } \quad+u \leq 0,
$$

which covers all of $\mathcal{P}^{\prime}$. Hence, the vertex $V[p]$ can be incident to the edge $E[r, q]$ only along the central plane $u=0$, and $\mathbf{H}_{a-b}$ is never needed to prevent overlaps within $\mathcal{P}^{\prime}$.

Lemma 4 The collection $\mathcal{P}^{\prime \prime}$ contains exactly two maximal density packings with density $\frac{4000}{4671}$. The packings are related by a crystallographic symmetry operation.

Proof The unit cell volume as a function of the parameters $\langle u, v, w\rangle$,

$$
V=|\operatorname{det}[a+b, b+c, c+a]|=\frac{9}{25}\left(117+60 u^{2}-80 u v-80 v^{2}\right),
$$

is a hyperbolic paraboloid with saddle point at $u=v=0$. The parameters $\langle u, v, w\rangle$ can be chosen such that $V$ does not depend on $w$, which means packings corresponding to the line $\langle 0,0, w\rangle$ are related by a lattice shear. Surfaces of equal volume are hyperbolic cylinders. The extrema of $\left.V\right|_{\mathcal{P}^{\prime \prime}}$ are taken on the boundary. We find that the densest packings are located on the maximal lines:

$$
\begin{array}{ll}
\mathbf{H}_{b+c} \wedge \mathbf{H}_{c-a}: & +\frac{3}{320}\langle 2,5,0\rangle+\frac{1}{6} u\langle 6,-1,+1\rangle, \\
\mathbf{H}_{b-c} \wedge \mathbf{H}_{c+a}: & -\frac{3}{320}\langle 2,5,0\rangle+\frac{1}{6} u\langle 6,-1,-1\rangle .
\end{array}
$$

Maximizing the packing density $\phi=2 U / V$ with the tetrahedron volume $U=$ $\frac{1}{6}|\operatorname{det}[o-p, o-q, o-r]|=9$ along the maximal lines yields $\phi=\frac{4000}{4671}$ and two optimal points:

$$
\left\langle u_{ \pm}, v_{ \pm}, w_{ \pm}\right\rangle= \pm \frac{3}{320}\langle 2,5,0\rangle .
$$

It can be shown that for two packings with parameters $\langle u, v, w\rangle$ and $\langle\bar{u}, \bar{v}, \bar{w}\rangle$ related by $\langle\bar{u}, \bar{v}, \bar{w}\rangle=\langle-u,-v, w\rangle$ there exists a direct isometry

$$
T=\frac{1}{3}\left(\begin{array}{lll}
+1 & -2 & -2 \\
-2 & -2 & +1 \\
-2 & +1 & -2
\end{array}\right)
$$


that symmetrically maps the lattice vectors $T a=-\bar{b}, T b=-\bar{a}, T c=-\bar{c}, T d=\bar{d}$ and the vertices of the dimers $T o=\bar{s}, T p=\bar{p}, T q=\bar{r}, T r=\bar{q}, T s=\bar{o}$. In particular, $T$ maps the optimal points onto each other.

\subsection{A Dense Double Dimer Packing}

The following theorem is the main result of our work. For the classification of crystallographic point groups and space groups we follow the terminology in [24].

Theorem 1 There exists a double dimer packing of tetrahedra with packing density $\frac{4000}{4671}$. Its space group is $\mathrm{P} \overline{1}$ (point group $\overline{1}$ ) and acts transitively on the dimers.

Proof The double dimer packing at the optimal point $\left\langle u_{+}, v_{+}, w_{+}\right\rangle$has density $\frac{4000}{4671}$. It is specified by the lattice vectors

$$
\begin{aligned}
a & =\frac{3}{320}\langle 290,107,-7\rangle, \\
b & =\frac{3}{320}\langle-34,277,135\rangle, \\
c & =\frac{3}{320}\langle 94,-83,247\rangle,
\end{aligned}
$$

and the offset vector

$$
d=\frac{1}{320}\langle 38,5,-25\rangle .
$$

We know from the angles between the lattice vectors that the crystal system is triclinic. The only non-translational symmetries are inversions mapping $\mathbf{L}^{+}$onto $\mathbf{L}^{-}$, which determines the space group.

Remark 2 (i) The lattice spanned by the vectors $a, b$, and $c$ in Theorem 1 can be obtained from a simple cubic lattice by a small deformation. Furthermore, the norm of $d$ is much smaller than the norms of $a, b, c$. These two observations mean that the set $\mathbf{L}=\mathbf{L}^{+} \cup \mathbf{L}^{-}$is structurally similar to the rock-salt lattice.

(ii) One of the axes that is almost a three-fold symmetry is the diagonal $a+b+c$, which coincides roughly with the dimer axis. By the choice of basis vectors in (15) and (16), the three-fold symmetry of the individual dimers is broken.

\subsection{Comparison with Previously Found Double Dimer Packings}

The one-parameter family of dense tetrahedron packings found by Kallus, Elser and Gravel [22] has constant packing density $\phi=\frac{100}{117}$ and corresponds to the symmetric line $\langle 0,0, w\rangle$. The packings are transitive on individual tetrahedra as a result of the symmetry $T$, a two-fold rotation around the axis through the vertex $p=\langle 2,-1,-1\rangle$.

Torquato-Jiao's two-parameter family of packings [23] corresponds to the generic plane $5 u=-2 v$. The intersections of this plane with the maximal lines gives the parameters $\frac{3}{2240}( \pm\langle 16,-40,0\rangle-\langle 0,0,5\rangle)$ and the maximum packing density $\phi=$ $\frac{12250}{14319}$. The two packings are not related by symmetry. 


\section{Numerical Compression of Small Cells}

\subsection{Motivation and Methods}

The packing given in Theorem 1 is optimal under two assumptions: (1) the densest tetrahedron packing is a double dimer packing, and (2) the packing is a configuration in the restricted parameter space $\mathcal{P}^{\prime \prime}$. To investigate the possibility of denser tetrahedron packings - double dimer packings as well as different types of packings-we rely on a numerical search.

We use standard Monte Carlo simulation [25], which allows a system of tetrahedra to find dense packings by stochastically exploring all possible configurations subject to the laws of statistical mechanics. An elementary simulation step consists of a random displacement move within a finite simulation cell- taking into account periodic boundary conditions - and a random rotation move of randomly chosen tetrahedra. The move is rejected if it generates an overlap, or accepted otherwise. The starting configuration is a dilute, random arrangement. During the simulation run, the system is slowly compressed by rescaling the size of the simulation cell. The compression is controlled by applying external forces in the isobaric ensemble. Additionally, fluctuations of cell shape by shearing the cell in random directions by random amounts are allowed. A lattice reduction technique minimizes the distortion of the simulation cell after each shear. For details of the tetrahedron overlap detection algorithm we refer to method two in the Methods section of [21].

The Monte Carlo scheme samples the high-dimensional configuration space stochastically, and is not biased towards any particular type of packing. The only constraint is the number of tetrahedra in the simulation cell, $N$, which does not change during the simulation. In the following, the search is restricted to small cells, $1 \leq N \leq 16$, where efficient compressions are easily and rapidly possible to high accuracy.

\subsection{Monte Carlo Simulation Results}

To obtain sufficient statistics, $M=400$ compression simulations were run for each value of $N$ (1000 runs for $N=16)$. Each run involves 7 million Monte Carlo moves per particle and results in a final density $\phi_{i}, i=1, \ldots, M$, for a given $N$. The maximum numerical density for a given $N$ is $\hat{\phi}=\max \left\{\phi_{i}\right\}$. The distribution of $\phi_{i}$ close to $\hat{\phi}$ indicates the ease with which we can obtain the optimal packing in simulation. For most $N$ we find a clear gap separating a set of very dense packings from the rest in the sense that the relative density variation among the very dense packings is significantly smaller than the gap between very dense and less dense packings. In this case, we denote the simulations corresponding to the very dense packings as successful.

The maximum numerical densities are given in Table 1 and their corresponding packings are depicted in Fig. 1. As can be seen from the success rates in the table, the geometrically constructed optimal packings for $N=1,2$ are obtained very easily and in every simulation. The $N=3$ packing is three-fold symmetric and, as far as we know, has not yet been reported in the literature. Its optimal density $\phi=\frac{2}{3}$ can be calculated analytically. The structures with $N=4,8,12,16$ are the dimer packings 
discussed in Sect. 2.4. The packing with $N=5$ consists of imperfect pentamers, i.e. four tetrahedra arranged face-to-face to a central one. $N=6$ is a mixture of dimers and single tetrahedra (monomers), and $N=7$ is identical to $N=8$ with one complete vacancy (missing tetrahedron).

For larger simulation cells crystalline packings are harder to achieve. The $N=10$ packing consists of two pentagonal dipyramids, perfect in the sense that four of each set of five tetrahedra are arranged face-to-face; the fifth tetrahedron is oriented in such a way to distribute the (obligatory) gap [15] evenly on its two sides. In the cases $N=9,11$, and 15 we found no clear density gap separating very dense packings from less dense packings. Either the density gap does not exist, or our simulations were not successful in finding the optimal packings. The latter must be the case for $N=15$, since a dimer packing with a vacancy can give a density of $\phi=\frac{3750}{4671}=0.802825 \ldots$ Instead, the compressions became trapped in disordered configurations with a network of pentagonal dipyramids, similar to that found in previous simulations [21]. It is noteworthy that all packings for $N \geq 4$ achieve a density $\phi>\phi_{\text {sphere }}$.

Table 1 Maximum numerical densities $\hat{\phi}$ for packings with small cells, obtained with numerical compression via Monte Carlo compression starting from a random configuration. A data file with the packings may be downloaded from the internet at [26]. For comparison, the quasicrystal approximant result with $N=$ $8 \times 82$ is included. Details about the analytical results $\phi_{2}=9 /(139-40 \sqrt{10}), \phi_{5}=0.74809657 \ldots, \phi_{6}=$ $11228544 /(97802181-132043 \sqrt{396129})$, and $\phi_{10}=29611698560 /(23657426736+4919428689 \sqrt{6})$ are given in Appendix D of [27]

\begin{tabular}{|c|c|c|c|c|}
\hline \multirow{2}{*}{$\begin{array}{c}\text { \#Tetra } \\
N\end{array}$} & \multicolumn{2}{|c|}{ Maximum density } & \multirow{2}{*}{$\begin{array}{r}\text { Success } \\
\text { rate }\end{array}$} & \multirow{2}{*}{$\begin{array}{l}\text { Motifs, } \\
\text { structural description }\end{array}$} \\
\hline & Numerical, $\hat{\phi}$ & Analytical, $\phi$ & & \\
\hline 1 & 0.367346 & $18 / 49$ & $100 \%$ & 1 monomer [11] \\
\hline 2 & 0.719486 & $\phi_{2}$ & $100 \%$ & 2 monomers, transitive [22] \\
\hline 3 & 0.666665 & $2 / 3$ & $21 \%$ & 3 monomers, three-fold symmetric \\
\hline 4 & 0.856347 & $4000 / 4671$ & $80 \%$ & 2 dimers (positive + negative) \\
\hline 5 & 0.748096 & $\phi_{5}$ & $22 \%$ & 1 pentamer, asymmetric \\
\hline 6 & 0.764058 & $\phi_{6}$ & $11 \%$ & 2 dimers +2 monomers \\
\hline 7 & 0.749304 & $3500 / 4671$ & $15 \%$ & $2 \times 2$ dimers minus 1 monomer \\
\hline 8 & 0.856347 & $4000 / 4671$ & $44 \%$ & $2 \times 2$ dimers, identical to $N=4$ \\
\hline 9 & 0.766081 & & - & 1 pentagonal dipyramid +2 dimers \\
\hline 10 & 0.829282 & $\phi_{10}$ & $2 \%$ & 2 pentagonal dipyramids \\
\hline 11 & 0.794604 & & - & 1 nonamer +2 monomers \\
\hline 12 & 0.856347 & $4000 / 4671$ & $3 \%$ & $3 \times 2$ dimers, identical to $N=4$ \\
\hline 13 & 0.788728 & & $4 \%$ & 1 pentagonal dipyramid +4 dimers \\
\hline 14 & 0.816834 & & $3 \%$ & 2 pentagonal dipyramids +2 dimers \\
\hline 15 & 0.788693 & & - & Disordered, non-optimal \\
\hline 16 & 0.856342 & $4000 / 4671$ & $<1 \%$ & $4 \times 2$ dimers, identical to $N=4$ \\
\hline$\vdots$ & $\vdots$ & & & $\vdots$ \\
\hline $8 \times 82$ & 0.850267 & & & Quasicrystal approximant [21] \\
\hline
\end{tabular}




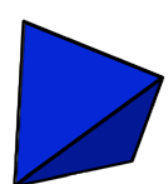

$N=1$

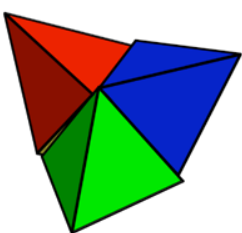

$N=5$

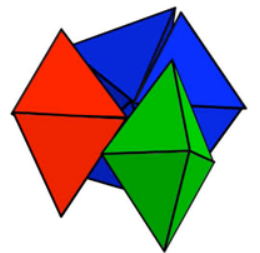

$N=9$

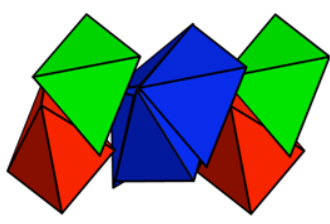

$N=13$

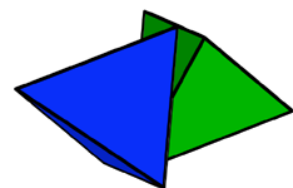

$N=2$

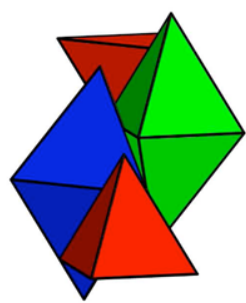

$N=6$

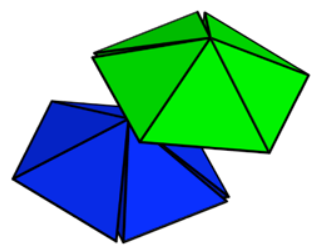

$N=10$

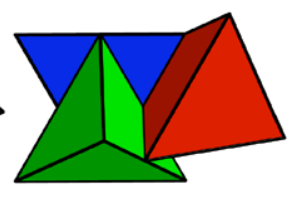

$N=3$
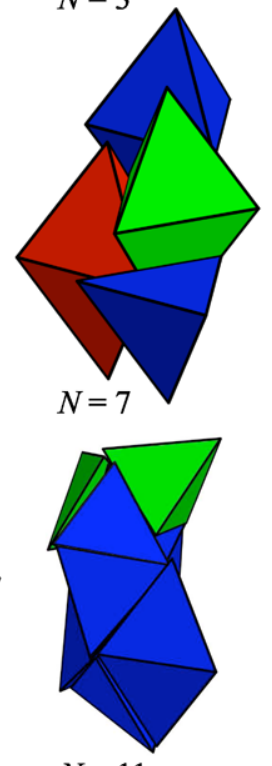

$N=11$
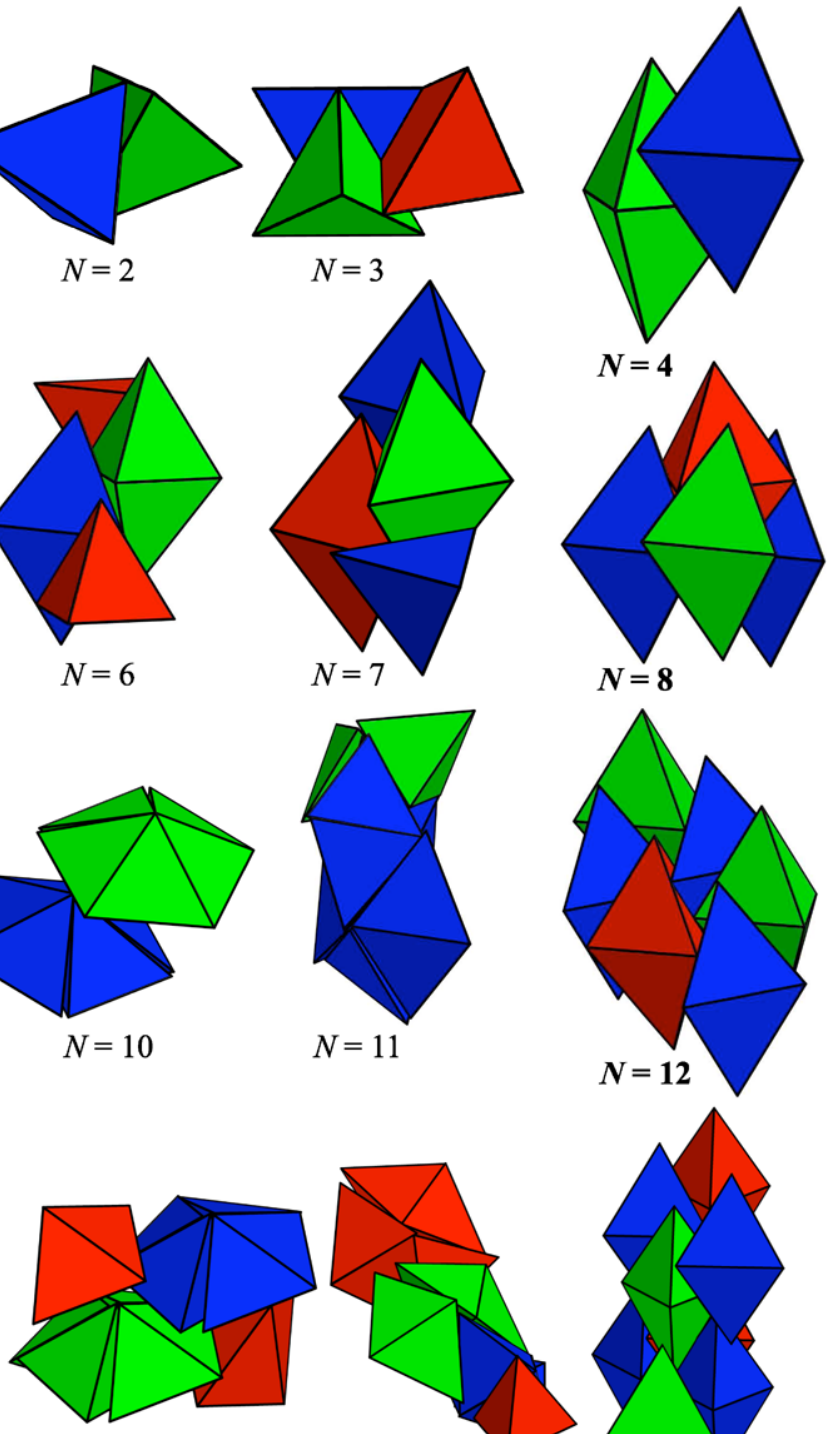

$N=14$

$$
N=15
$$

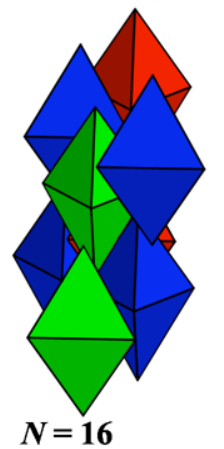

Fig. 1 (Color online) Dense packings with up to 16 tetrahedra per cell as obtained from Monte Carlo simulations. The densest dimer packing is observed for $N=4,8,12,16$ 


\section{Conclusions}

We have found the densest known packing of tetrahedra with density $\phi=\frac{4000}{4671}$. This result was obtained as the optimal solution of a three-parameter family of dimer packings, which is a generalization of one-parameter and two-parameter families of packings recently reported with lower maximum densities. Interestingly, the densest packing is not the most symmetric one. Isobaric Monte Carlo simulations with variable cell shape starting from random initial conditions recover the same high packing density within $10^{-5}$ for small systems containing 4, 8, 12, and 16 tetrahedra. We also discovered new candidates for densest packings with $3,5,6$, and 10 tetrahedra per unit cell. The analytical and numerical results combined suggest that the packing density reported here could be the highest achievable, at least for small $N$.

The dimer structures are remarkable in the relative simplicity of the 4-tetrahedron unit cell as compared to the 82-tetrahedron unit cell of the quasicrystal approximant [21], whose density is only slightly less than that of the densest dimer packing. The dodecagonal quasicrystal is the only ordered phase observed to form from random initial configurations of large collections of tetrahedra at moderate densities. It is thus interesting to note that for some certain values of $N$, when the small systems do not form the dimer lattice packing, they instead prefer clusters (motifs) present in the quasicrystal and its approximant, predominantly pentagonal dipyramids. This suggests that the two types of packings - the dimer crystal and the quasicrystal/approximantmay compete, raising interesting questions about the relative stability of the two very different structures at finite pressure. These questions will be explored in a forthcoming paper.

Acknowledgements E.R.C. is grateful to Jeffrey C. Lagarias for his many efforts to make this research possible. We are very grateful to him and to the anonymous referees for their helpful comments on the manuscript.

E.R.C. acknowledges a National Science Foundation RTG grant DMS-0801029. M.E. acknowledges the support of a postdoctoral fellowship from the Deutsche Forschungsgemeinschaft (EN 905/1-1). S.C.G. acknowledges support from the Air Force Office of Scientific Research under MURI grant FA9550-06-10337.

\section{References}

1. Tang, Z.Y., Zhang, Z.L., Wang, Y., Glotzer, S.C., Kotov, N.A.: Spontaneous self-assembly of CdTe nanocrystals into free-floating sheets. Science 314, 274-278 (2006)

2. Zhang, Z.-L., Tang, Z.-Y., Kotov, N.A., Glotzer, S.C.: Self-assembly of CdTe nanoparticles into wires and sheets. Nano Letters 7, 1670-1675 (2007)

3. Srivastava, S., Santos, A., Critchley, K., Kim, K.-S., Podsiadlo, P., Sun, K., Lee, J., Lilly, G.D., Glotzer, S.C., Kotov, N.A.: Light-controlled self-assembly of semi-conductor nanoparticles into twisted ribbons. Science 327, 1355-1359 (2010)

4. Chen, E.R.: A picturebook of tetrahedral packings. Ph.D. Dissertation, University of Michigan (2010) 
5. Struik, D.J.: De impletione loci. Nieuw Arch. Wiskd. 15, 121-134 (1925)

6. Senechal, M.: Which tetrahedra fill space? Math. Mag. 54, 227-243 (1981)

7. Minkowski, H.: Geometrie der Zahlen. Teubner, Leipzig (1896)

8. Minkowski, H.: Dichteste gitterförmige Lagerung kongruenter Körper. Nachr. Ges. Wiss. Gött. 311355 (1904)

9. Hilbert, D.C.: Mathematische Probleme. Nachr. Ges. Wiss. Gött., Math.-Phys. Kl. 3, 253-297 (1900)

10. Grömer, H.: Über die dichteste gitterförmige Lagerung kongruenter Tetraeder. Monatshefte Math. 66, $12-15(1962)$

11. Hoylman, D.J.: The densest lattice packing of tetrahedra. Bull. Am. Math. Soc. 76, 135-137 (1970)

12. Gardner, M.: The Colossal Book of Mathematics: Classic Puzzles, Paradoxes, and Problems. Norton, New York (2001), $135 \mathrm{pp}$.

13. Hales, T.C.: A proof of the Kepler conjecture. Ann. Math. 162, 1065-1185 (2005)

14. Betke, U., Henk, M.: Densest lattice packings of 3-polytopes. Comput. Geom. 16, 157-186 (2000)

15. Conway, J.H., Torquato, S.: Packing, tiling and covering with tetrahedra. Proc. Natl. Acad. Sci. USA 103, 10612-10617 (2006)

16. Chaikin, P., Wang, S., Jaoshvili, A.: Packing of tetrahedral and other dice. In: American Physical Society March Meeting, Denver, CO, paper S29.00010, 2007

17. Jaoshvili, A., Esakia, A., Porrati, M., Chaikin, P.M.: Experiments on the random packing of tetrahedral dice. Phys. Rev. Lett. 104, 185501 (2010). doi:10.1103/PhysRevLett.104.185501

18. Chen, E.R.: A dense packing of regular tetrahedra. Discrete Comput. Geom. 40, 214-240 (2008)

19. Haji-Akbari, A., Engel, M., Keys, A.S., Zheng, X.Y., Petschek, R., Palffy-Muhoray, P., Glotzer, S.C.: Dense packings of hard tetrahedra. In: American Physical Society March Meeting, Pittsburgh, PA, paper X9.00009, 2009

20. Torquato, S., Jiao, Y.: Dense packings of the Platonic and Archimedean solids. Nature 460, 876-879 (2009)

21. Haji-Akbari, A., Engel, M., Keys, A.S., Zheng, X.Y., Petschek, R.G., Palffy-Muhoray, P., Glotzer, S.C.: Disordered, quasicrystalline, and crystalline phases of densely packed tetrahedra. Nature $\mathbf{4 6 2}$, 773-777 (2009)

22. Kallus, Y., Elser, V., Gravel, S.: Dense periodic packings of tetrahedra with a small repeating units. Discrete Comput. Geom. (2010). doi:10.1007/s00454-010-9254-3. arXiv:0910.5226 (2009)

23. Torquato, S., Jiao, Y.: Analytical constructions of a family of dense tetrahedron packings and the role of symmetry. arXiv:0912.4210v3 (2009)

24. Hahn, T. (ed.): International Tables for Crystallography, Volume A: Space Group Symmetry, 5th edn. Springer, Berlin (2002)

25. Frenkel, D., Smit, B.: Understanding Molecular Simulation: From Algorithms to Applications. Academic Press, New York (2001)

26. http://glotzerlab.engin.umich.edu/wiki/public/index.php/Tetrahedra

27. Chen, E.R., Engel, M., Glotzer, S.C.: Dense crystalline dimer packings of regular tetrahedra. Supplementary info in arXiv:1001.0586 (2010)

\section{Appendix A: Pictures and Equations}

This appendix contains figures of the double dimer packings (Figs. A1-A3), the three-dimensional restricted parameter space (Fig. A4), and the volume contour function (Fig. A5), as well as visualizations of the internal symmetry of the restricted parameter space (Figs. A6 and A7). 
Figure A0 introduces the orientation of the axes for all following figures. We distinguish between the vector coordinate axes $\langle x, y, z\rangle$ and the packing coordinate axes $\langle u, v, w\rangle$. In the former case, the viewpoints are $\langle 1,1,1\rangle,\left\langle 0,-\cos \frac{\pi}{10}, \sin \frac{\pi}{10}\right\rangle$, $\langle 0,0,1\rangle$, and the ranges are $-\frac{32}{5} \leq x, y, z \leq+\frac{32}{5}$ (Figs. A1-A3) or $-\frac{15}{4} \leq x, y, z \leq$ $+\frac{15}{4}$ (Fig. A6). In the latter case, the viewpoints are $\left\langle\cos \frac{2 \pi}{15} \cos \frac{\pi}{15}, \cos \frac{2 \pi}{15} \sin \frac{\pi}{15}\right.$, $\left.\sin \frac{2 \pi}{15}\right\rangle,\left\langle\sin \frac{\pi}{120}, 0, \cos \frac{\pi}{120}\right\rangle$, and the range is $-\frac{1}{8} \leq u, v, w \leq+\frac{1}{8}$ (Figs. A4-A5 and A7).

Figure A1 (4 pages) gives the notation and basic equations, and shows the nearest intersecting neighbors. The colors of the equations match the color of the respective neighbors. Since the double dimer packings are cluster transitive on dimers, we consider only the dimer at the origin. Its positive and negative neighbors are shown for three pairs of special packings at the optimal points, the central points, and the symmetric points in parameter space (Fig. A4). The pairs of packings are related by the symmetry $T$ (Fig. A7). Notice that the sets of negative neighbors are always the same, whereas the sets of positive neighbors are different. The neighbors for the optimal packings are related by a reflection through the plane $+2 x-y-z=0$, the neighbors for the central packings by a reflection through the plane $+2 x-y-z=0$, and the neighbors for the symmetric packings by a reflection through the plane which contains $\pm a \pm b$.

Figure A2 (2 pages) lists all 20 possible neighbors that can intersect with the dimer at the origin together with their linear incidence conditions. The entire figure is a floor plan diagram of the neighbors, ordered by layer. Notice how the positive layers and the negative layers complement each other. The even layers are related by symmetry, which is broken for the odd layers due to the presence of a small translation by the vector $d$.

Figure A3 (2 pages) shows larger portions of the layers for the optimal packings. The entire figure is a floor plan diagram of the packing, ordered by layer. Notice the almost triangular/hexagonal planar basis mentioned in Remark 2. We show both optimal packings for comparison to demonstrate the subtle differences. The symmetry $T$ that maps the packings onto each other is a two-fold rotation around an axis running from bottom left to top right in the left columns (view along the 'almost' three-fold axis).

Figure A4 (1 page) depicts the restricted three-dimensional parameter space of dense double dimer packings $\langle u, v, w\rangle$ together with special planes, lines, and points. The shape is a strongly deformed tetrahedron extended along the $u$-axis. It is bounded by four boundary planes. Packings with maximal (minimal) density are located on the maximal (minimal) lines (Fig. A5). The optimal plane contains the two optimal points, the origin, and the symmetric line.

Figure A5 (1 page) visualizes contours of the unit cell volume $V=\frac{9}{25}\left(117+60 u^{2}-\right.$ $\left.80 u v-80 v^{2}\right)$ in parameter space. The lattice volume is a hyperbolic paraboloid in $\langle u, v, V\rangle$. The contours are hyperbolic cylinders in $\langle u, v, w\rangle$ parallel to the $w$-axis. Their intersection with the restricted parameter space (gray) allows to identify the locations of volume extrema on the maximal and minimal lines. 
Figure A6 (2 pages) demonstrates the distortion in the geometry of nearest neighbors in vector space $\langle x, y, z\rangle$. The colored spheres (colors match the colors in Figs. A1A3) are positioned at the centers of the twelve dimers in the layer $0, \pm 1$, closest to the dimer at the origin. Lines connect dimers that are adjacent in the dimer lattice. A deformation of the neighbor shell occurs when moving along the various lines in parameter space $\langle u, v, w\rangle$. This deformation is illustrated in each sub-figure by overlaying three neighbor configurations along these lines. Packings along the central line have the symmetry $T$, which is a two-fold rotation about the axis $p=\langle+2,-1,-1\rangle$ (see proof of Theorem 2). Notice how these packings and distortions look more symmetric than the others. Packings along the maximal lines form a double family. Packings along the minimal lines form two single families that are distorted in complementary ways.

Figure A7 (1 page) illustrates the effect of the symmetry $T$ on the restricted parameter space shown in Fig. A4 by identifying the equivalent packings under this symmetry: $T\langle u, v, w\rangle=\langle-u,-v, w\rangle$. Within contour planes of the form $w=*$, i.e. normal to the $w$-axis, $T$ acts as a combination of a shear and a two-fold rotation around the symmetric line $\langle 0,0, w\rangle$. Equivalent points are colored identically. Along the minimal lines, opposite points on the same line are related. Notice that each minimal line is contained in a single contour plane. Along the maximal lines, opposite points on opposite lines are related. Notice that the two optimal points are contained in a single contour plane. The final sub-figure shows the change of the contour plane intersection with the maximal lines for a sequence of five contour planes.
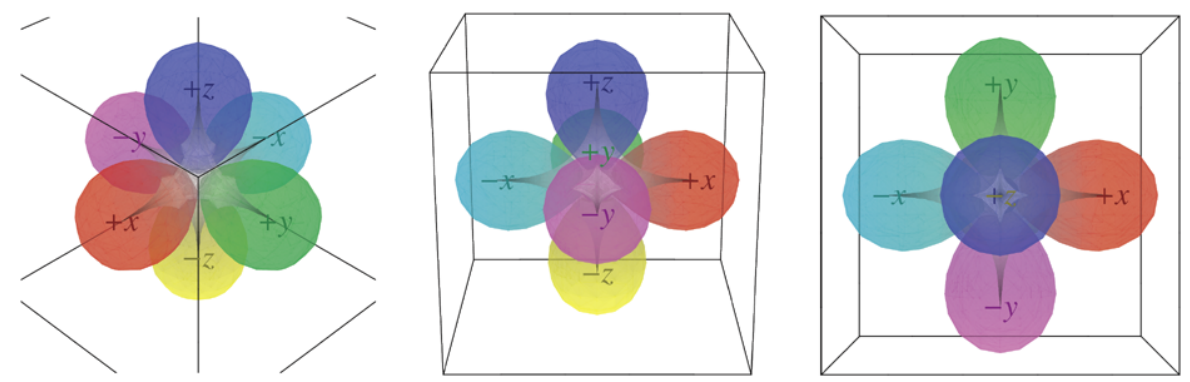

Fig. $\mathbf{A} \mathbf{0}_{\mathrm{vec}}$ The vector $\langle x, y, z\rangle$ coordinate axes for Figs. A1-A3 and A6

Fig. $\mathbf{A} \mathbf{0}_{\text {pac }}$ The packing $\langle u, v, w\rangle$ coordinate axes for Figs. A4-A5 and A7
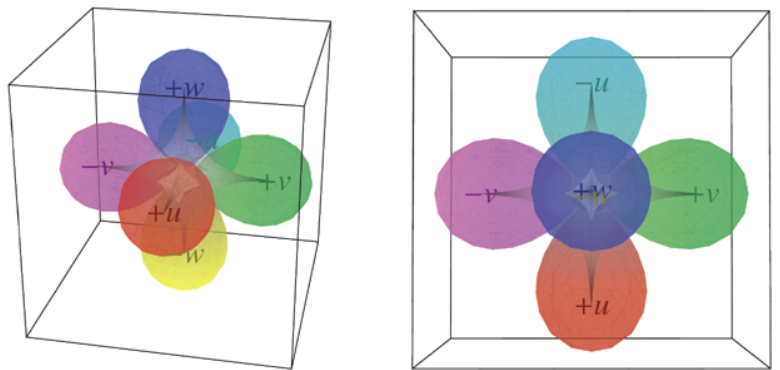
The cluster $\mathbf{F}_{2}$ has vertices

$$
\begin{aligned}
& o=\langle+2,+2,+2\rangle \\
& p=\langle+2,-1,-1\rangle \\
& q=\langle-1,+2,-1\rangle \\
& r=\langle-1,-1,+2\rangle \\
& s=\langle-2,-2,-2\rangle
\end{aligned}
$$

The cluster volume is

$$
U=\frac{1}{6} \operatorname{det}[o-p, o-q, o-r]+\frac{1}{6} \operatorname{det}[s-p, s-q, s-r]=18
$$

Clusters have 2 possible orientations

$+\mathbf{F}_{2}$ positive

$-\mathbf{F}_{2}$ negative (point reflection $=$ scalar multiplication by -1 )

The family of packings is parametized by

$$
\left\langle a_{x}, b_{y}, c_{z}\right\rangle=\left\langle+\frac{27}{10},+\frac{51}{20},+\frac{753}{320}\right\rangle+\left\langle u, v, \frac{1}{2} u-v+w\right\rangle
$$

The lattice vectors are

$$
\begin{aligned}
& a=\left\langle a_{x}, a_{y}, a_{z}\right\rangle=\left\langle+\frac{27}{10},+\frac{21}{20},-\frac{3}{20}\right\rangle+u\langle 1,0,2\rangle-v\langle 0,+1,-1\rangle \\
& b=\left\langle b_{x}, b_{y}, b_{z}\right\rangle=\left\langle-\frac{3}{10},+\frac{51}{20},+\frac{27}{20}\right\rangle-u\langle 1,0,2\rangle+v\langle 0,+1,-1\rangle \\
& c=\left\langle c_{x}, c_{y}, c_{z}\right\rangle=\left\langle+\frac{129}{160},-\frac{237}{320},+\frac{753}{320}\right\rangle-\left(\frac{1}{2} u-v\right)\langle+2,-1,-1\rangle+w\langle 2,3,1\rangle \\
& d=\left\langle d_{x}, d_{y}, d_{z}\right\rangle=\left\langle+\frac{1}{10},-\frac{1}{20},-\frac{1}{20}\right\rangle+u\langle 1,1,1\rangle+v\langle 0,+1,-1\rangle
\end{aligned}
$$

The lattice volume and packing density are

$$
\begin{aligned}
& V=2 \operatorname{det}[a, b, c]=\operatorname{det}[a+b, b+c, c+a] \\
& \phi=2 U / V=36 / V
\end{aligned}
$$
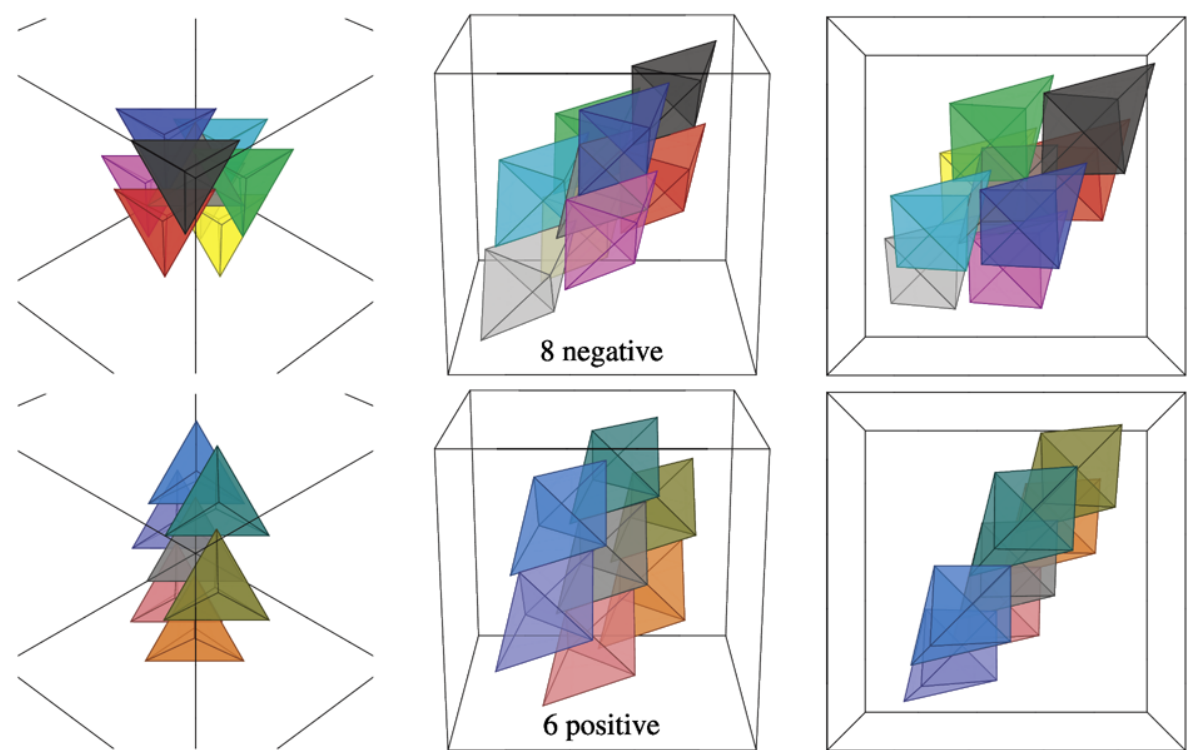

Fig. A1 $1_{\text {opt }}^{+}$The 14 intersecting neighbors of the optimal packing $\langle u, v, w\rangle=\left\langle+\frac{3}{160},+\frac{3}{64}, 0\right\rangle$ 
The cluster $+\mathbf{F}_{2}$ has 20 neighbors in various layers

The 12 positive neighbors are

$$
\begin{array}{rrrr}
+\mathbf{F}_{2}+a+b & +\mathbf{F}_{2}+b+c & +\mathbf{F}_{2}+c+a & +2 \text { layer } \\
+\mathbf{F}_{2}+a-b & +\mathbf{F}_{2}+b-c & +\mathbf{F}_{2}+c-a & 0 \text { layer } \\
+\mathbf{F}_{2}-a+b & +\mathbf{F}_{2}-b+c & +\mathbf{F}_{2}-c+a & 0 \text { layer } \\
+\mathbf{F}_{2}-a-b & +\mathbf{F}_{2}-b-c & +\mathbf{F}_{2}-c-a & -2 \text { layer }
\end{array}
$$

The 8 negative neighbors are

$$
\begin{array}{cccc} 
& -\mathbf{F}_{2}+d+a+b+c & & +3 \text { layer } \\
-\mathbf{F}_{2}+d+a & -\mathbf{F}_{2}+d+b & -\mathbf{F}_{2}+d+c & +1 \text { layer } \\
-\mathbf{F}_{2}+d-a & -\mathbf{F}_{2}+d-b & -\mathbf{F}_{2}+d-c & -1 \text { layer } \\
& -\mathbf{F}_{2}+d-a-b-c & & -3 \text { layer }
\end{array}
$$

The 2 optimal packings are parametrized by

$$
\langle u, v, w\rangle=\left\langle+\frac{3}{160},+\frac{3}{64}, 0\right\rangle \quad\langle u, v, w\rangle=\left\langle-\frac{3}{160},-\frac{3}{64}, 0\right\rangle
$$

The lattice vectors are

$$
\begin{aligned}
& a=\left\langle+\frac{87}{32},+\frac{321}{320},-\frac{21}{320}\right\rangle \\
& b=\left\langle-\frac{51}{160},+\frac{831}{320},+\frac{81}{64}\right\rangle \\
& c=\left\langle+\frac{141}{16},-\frac{249}{320},+\frac{741}{320}\right\rangle \\
& d=\left\langle+\frac{19}{160},+\frac{1}{64},-\frac{5}{64}\right\rangle
\end{aligned}
$$

$a=\left\langle+\frac{429}{160},+\frac{351}{320},-\frac{15}{64}\right\rangle$

$b=\left\langle-\frac{9}{32},+\frac{801}{320},+\frac{459}{320}\right\rangle$

$c=\left\langle+\frac{117}{160},-\frac{45}{64},+\frac{153}{64}\right\rangle$

$d=\left\langle+\frac{13}{160},-\frac{37}{320},-\frac{7}{320}\right\rangle$

The lattice volume and packing density are
$V=\frac{42039}{1000}$
$V=\frac{42039}{1000}$
$\phi=\frac{4000}{4671}$
$\phi=\frac{4000}{4671}$
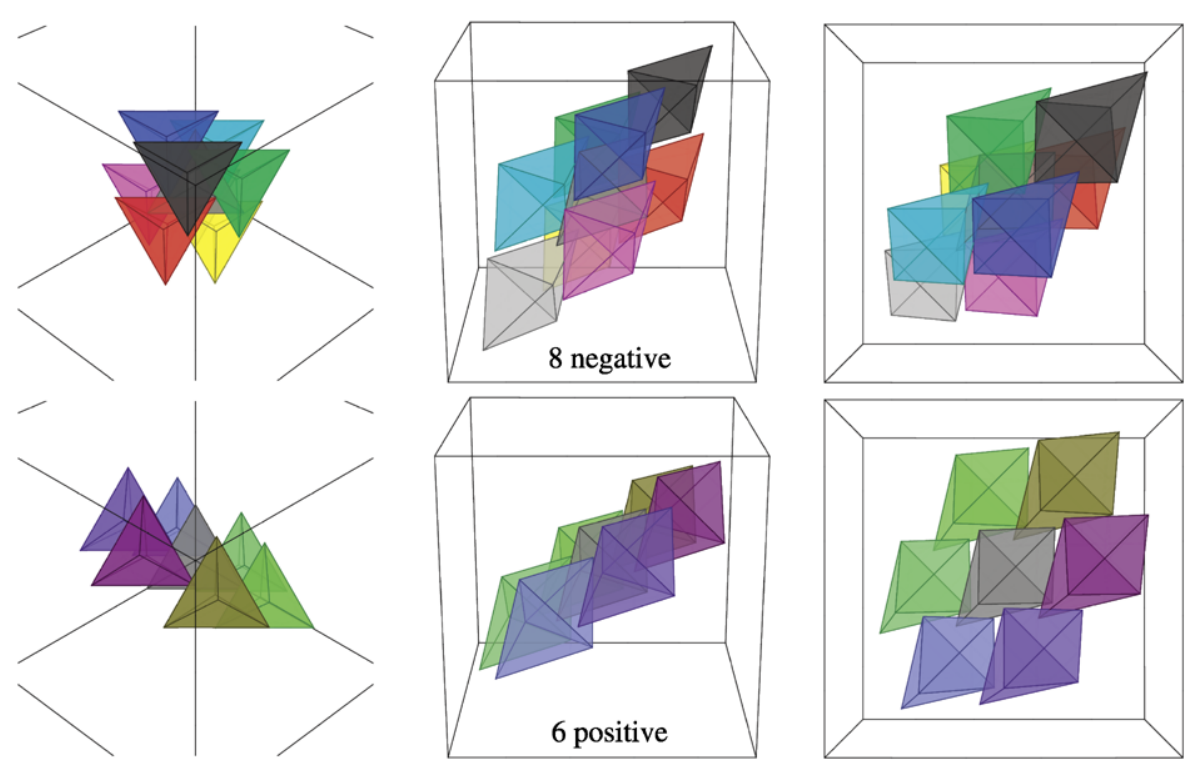

Fig. $\mathbf{A} 1_{\text {opt }}^{-}$The 14 intersecting neighbors of the optimal packing $\langle u, v, w\rangle=\left\langle-\frac{3}{160},-\frac{3}{64}, 0\right\rangle$ 

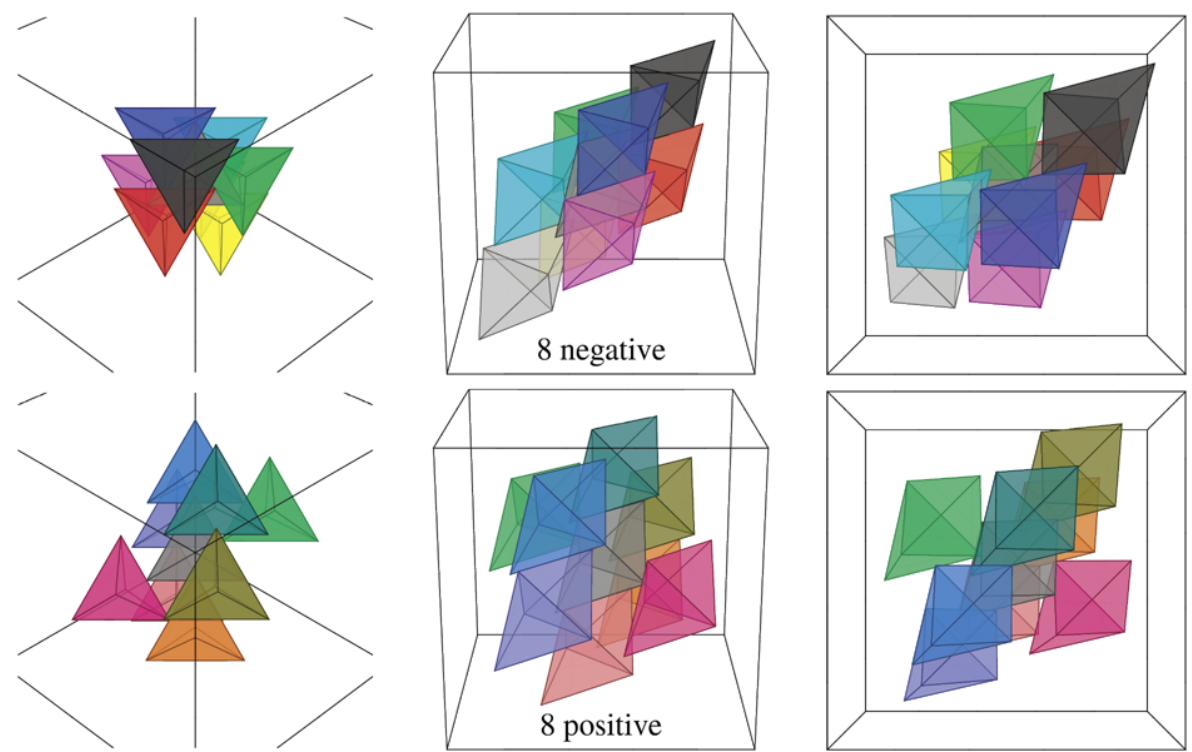

Fig. $\mathbf{A} \mathbf{1}_{\text {cen }}^{+}$The 16 intersecting neighbors of the central packing $\langle u, v, w\rangle=\left\langle 0,+\frac{1}{20},-\frac{1}{320}\right\rangle$
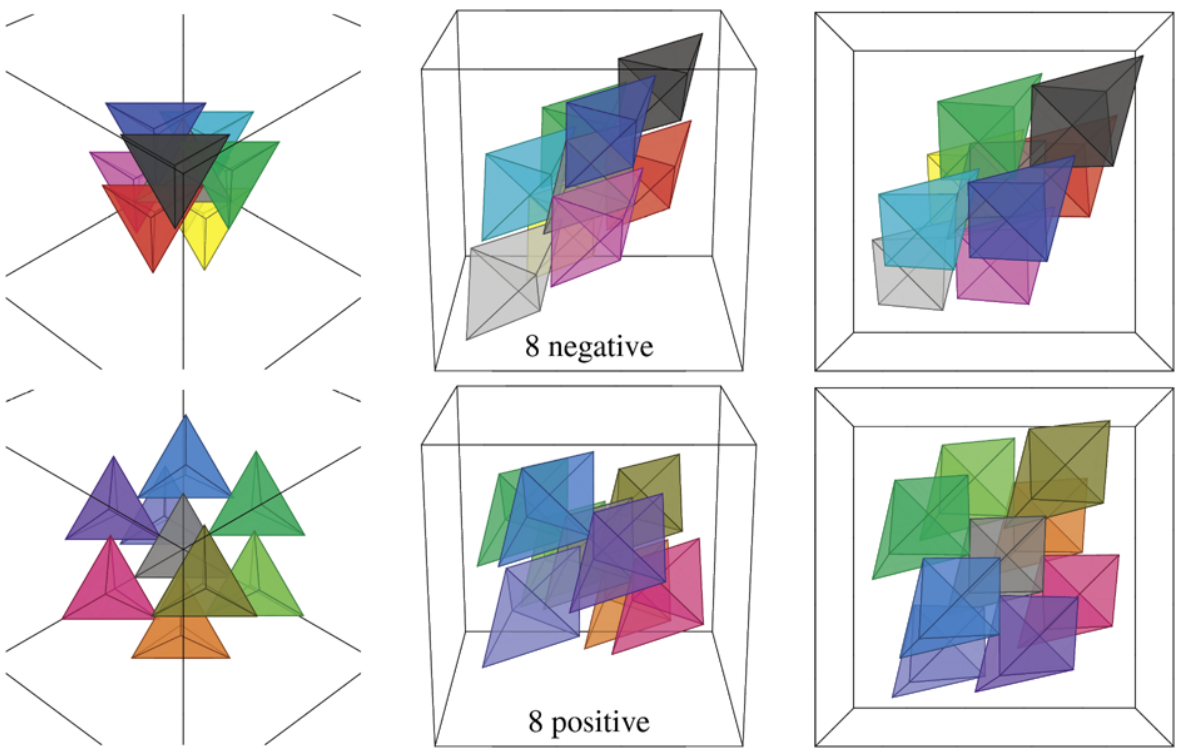

Fig. $\mathbf{A} \mathbf{1}_{\text {sym }}^{+}$The 16 intersecting neighbors of the symmetric packing $\langle u, v, w\rangle=\left\langle 0,0,+\frac{3}{64}\right\rangle$ 

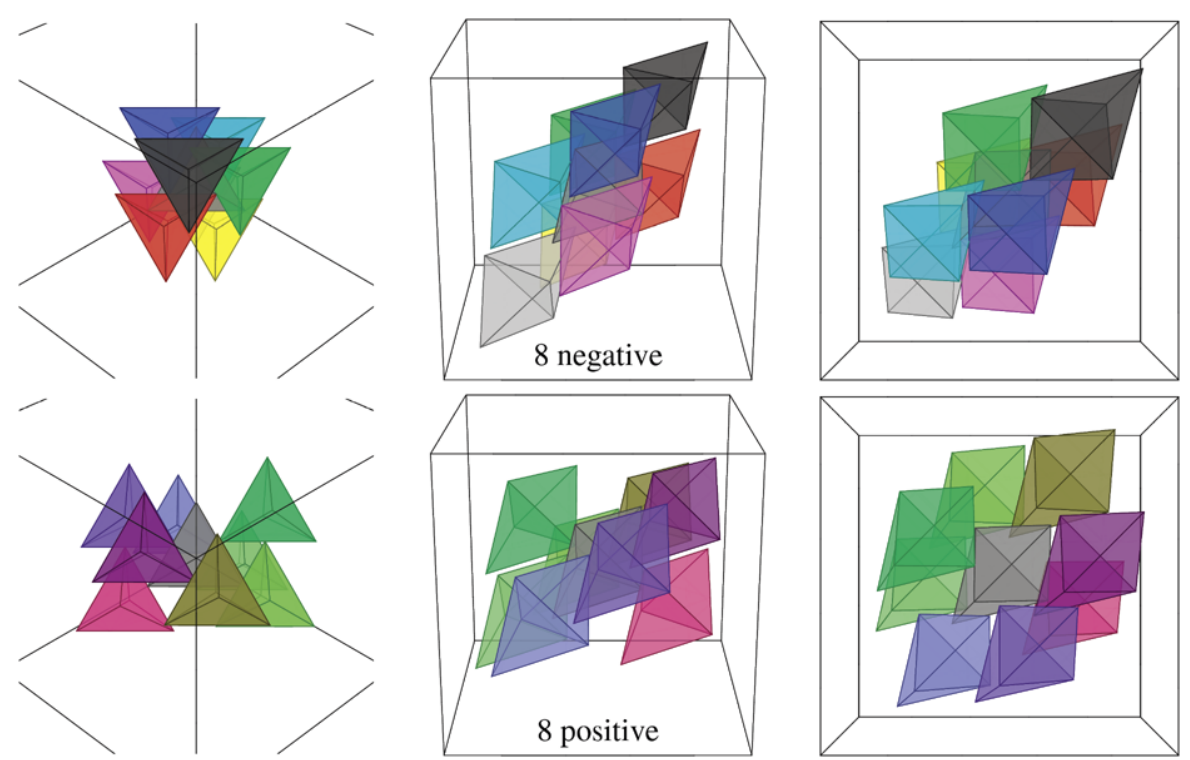

Fig. $\mathbf{A} \mathbf{1}_{\text {cen }}^{-}$The 16 intersecting neighbors of the central packing $\langle u, v, w\rangle=\left\langle 0,-\frac{1}{20},-\frac{1}{320}\right\rangle$
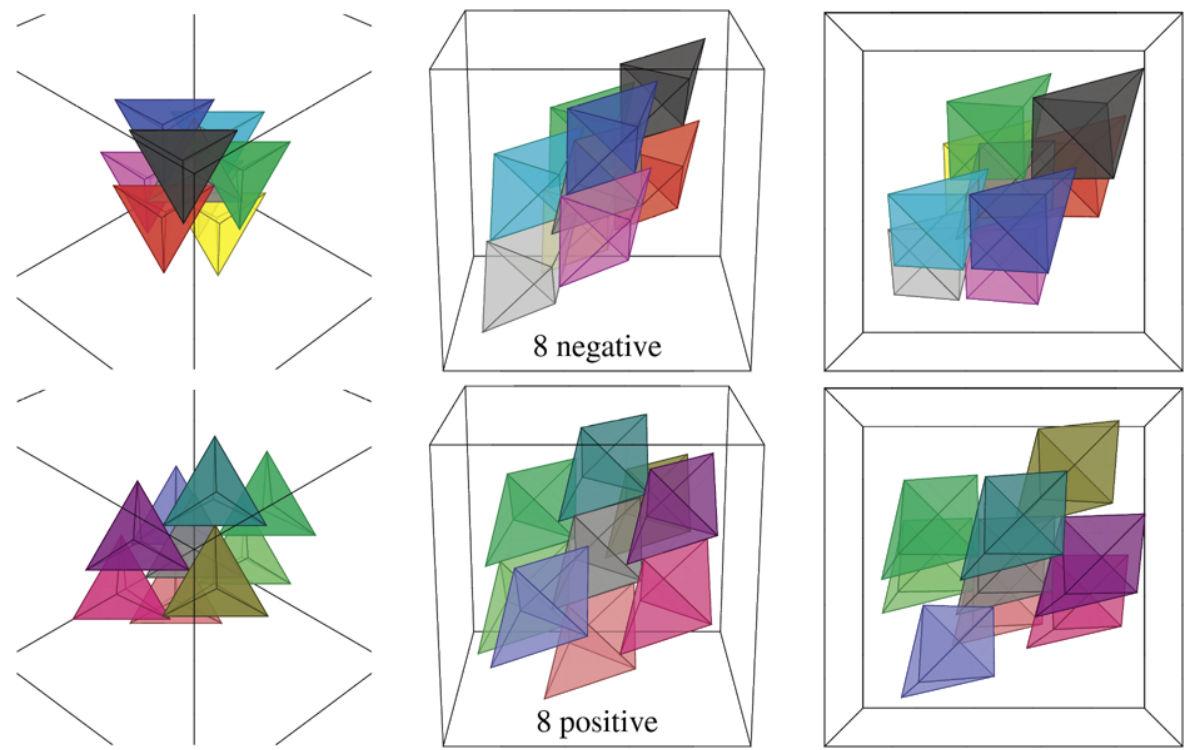

Fig. $\mathbf{A} \mathbf{1}_{\text {sym }}^{-}$The 16 intersecting neighbors of the symmetric packing $\langle u, v, w\rangle=\left\langle 0,0,-\frac{33}{320}\right\rangle$ 


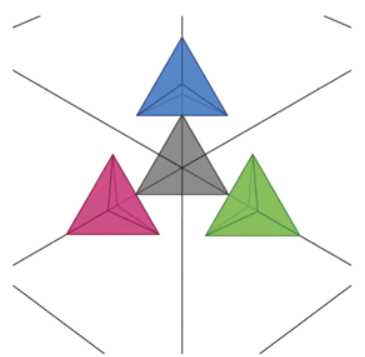

$\mathbf{H}_{a-b} \quad V[p] \cap E[r, q]+a-b$

$\mathbf{H}_{b-c} \quad V[q] \cap F[s, p, r]+b-c$

$\mathbf{H}_{c-a} \quad V[r] \cap F[o, q, p]+c-a$

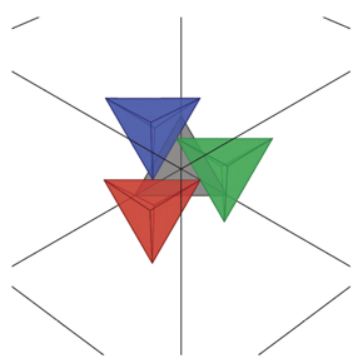

$\mathbf{G}_{a}^{+} \quad F[o, p, q] \cap d-F[o, p, q]+a$

$\mathbf{G}_{b}^{+} \quad F[o, q, r] \cap d-F[o, q, r]+b$

$\mathbf{G}_{c}^{+} \quad F[o, r, p] \cap d-F[o, r, p]+c$

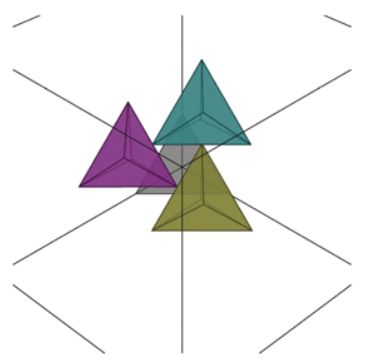

$\mathbf{G}_{a+b} \quad E[o, q] \cap E[s, r]+a+b$

$\mathbf{H}_{b+c} \quad E[o, r] \cap E[s, p]+b+c$

$\mathbf{H}_{c+a} \quad E[o, p] \cap E[s, q]+c+a$

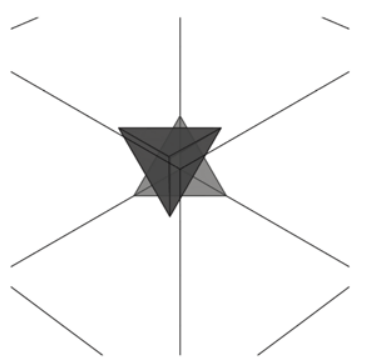

$\mathbf{G}_{a+b+c}^{+} F[o, r, p] \cap d-F[o, r, p]+a+b+c$
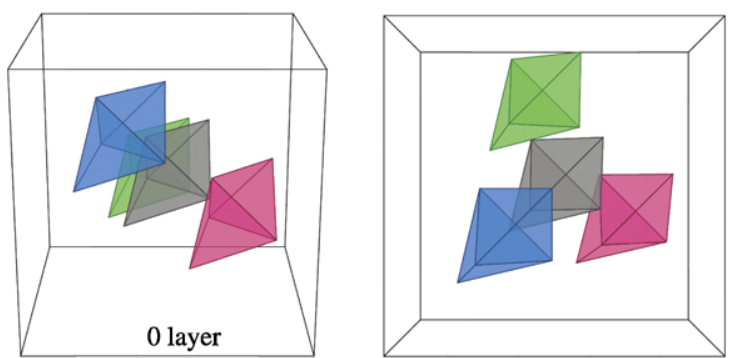

$\mathbf{F}_{2} \cap \mathbf{F}_{2}+a-b \quad+x-y-z=4$

$+5 x-y-z=12$

$\mathbf{F}_{2} \cap \mathbf{F}_{2}+b-c$

$-x+5 y-z=12$

$\mathbf{F}_{2} \cap \mathbf{F}_{2}+c-a$

$-x-y+z=4$

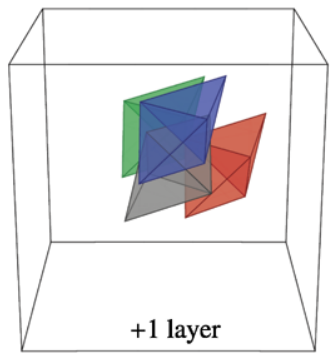

$\mathbf{F}_{2} \cap d-\mathbf{F}_{2}+a$

$\mathbf{F}_{2} \cap d-\mathbf{F}_{2}+b$

$\mathbf{F}_{2} \cap d-\mathbf{F}_{2}+c$

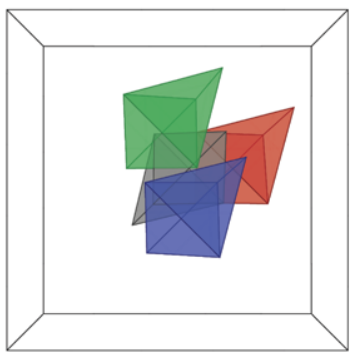

$+x+y-z=2$

$-x+y+z=2$

$+x-y+z=2$

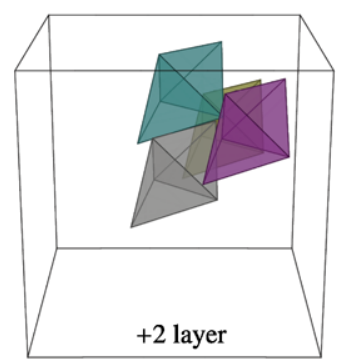

$\mathbf{F}_{2} \cap \mathbf{F}_{2}+a+b$

$\mathbf{F}_{2} \cap \mathbf{F}_{2}+b+c$

$\mathbf{F}_{2} \cap \mathbf{F}_{2}+c+a$

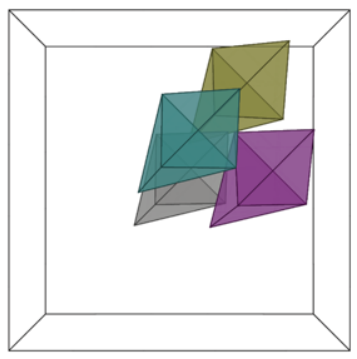

$+x+3 y-z=6$

$-x+y+3 z=6$

$+3 x-y+z=6$

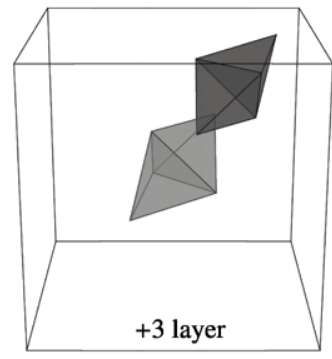

$\mathbf{F}_{2} \bigcap d-\mathbf{F}_{2}+a+b+c$

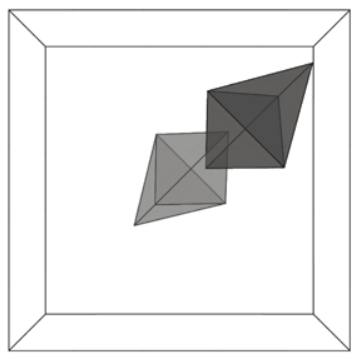

$+x-y+z=2$

Fig. A2 ${ }^{+}$The neighbors, intersection equations and separating planes of $\mathbf{F}_{2}$ (positive layers) 

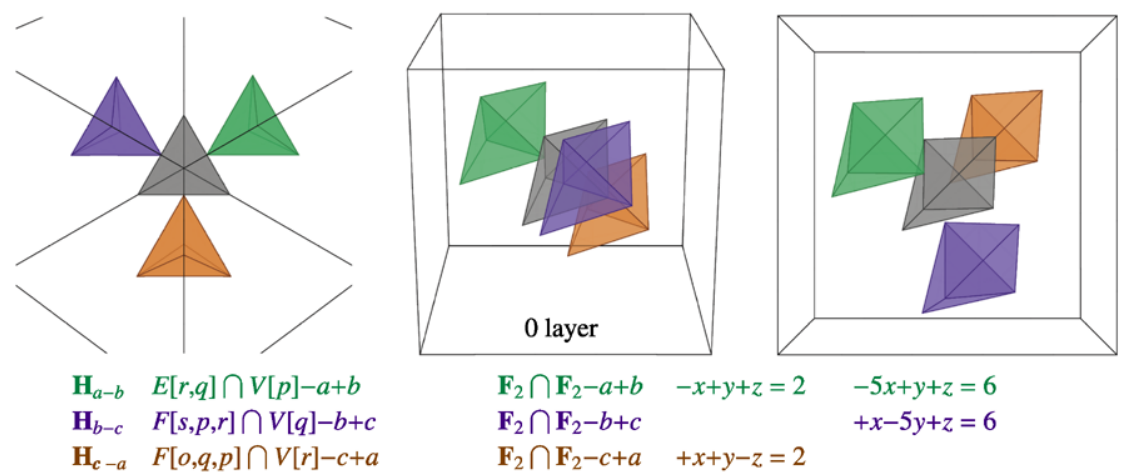

$$
\begin{aligned}
& \mathbf{F}_{2} \cap \mathbf{F}_{2}-a+b \quad-x+y+z=2 \quad-5 x+y+z=6 \\
& \mathbf{F}_{2} \cap \mathbf{F}_{2}-b+c \quad+x-5 y+z=6
\end{aligned}
$$

$\mathbf{H}_{c-a} \quad F[o, q, p] \cap V[r]-c+a$
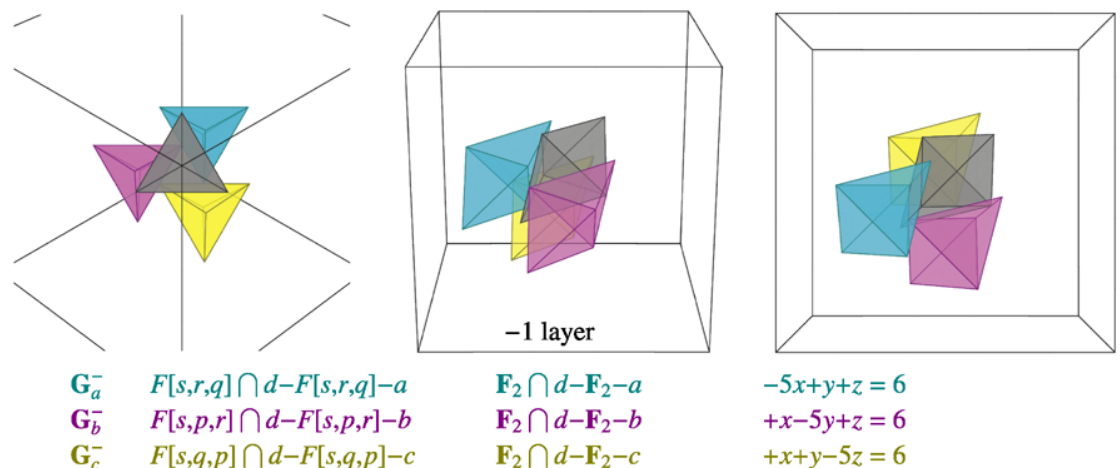

$$
\begin{aligned}
& \mathbf{F}_{2} \bigcap d-\mathbf{F}_{2}-a \\
& \mathbf{F}_{2} \bigcap d-\mathbf{F}_{2}-b
\end{aligned}
$$$$
-5 x+y+z=6
$$

$$
+x-5 y+z=6
$$$$
+x+y-5 z=6
$$
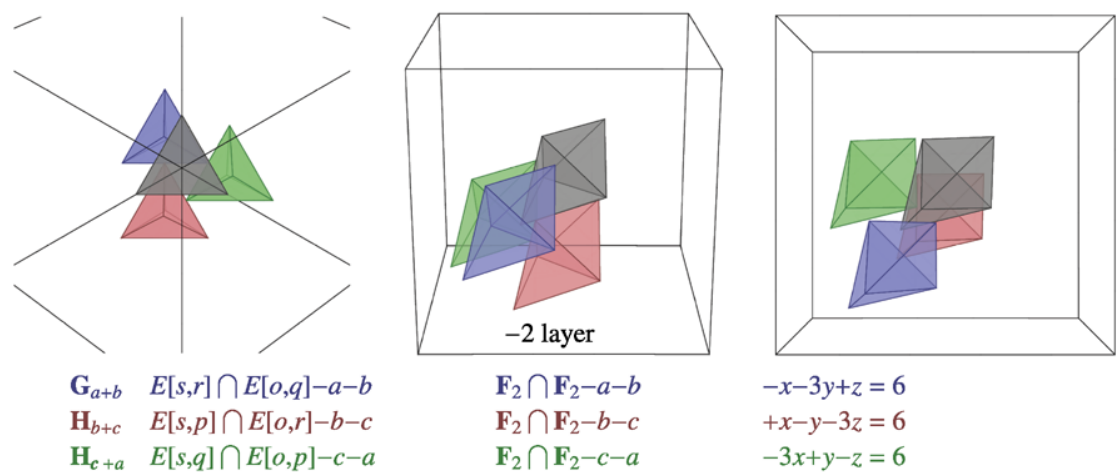

$\mathbf{F}_{2} \cap \mathbf{F}_{2}-a-b$

$$
-x-3 y+z=6
$$

$\mathbf{F}_{2} \cap \mathbf{F}_{2}-b-c$

$+x-y-3 z=6$

$\mathbf{H}_{c+a} \quad E[s, q] \cap E[o, p]-c-a$

$\mathbf{F}_{2} \cap \mathbf{F}_{2}-c-a$

$-3 x+y-z=6$
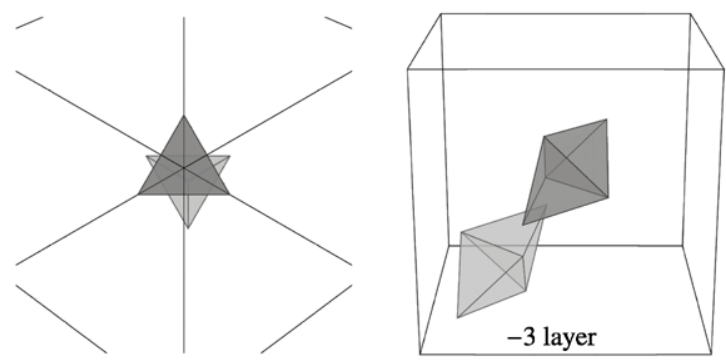

$\mathbf{G}_{a+b+c}^{-} F[s, q, p] \cap d-F[s, q, p]-a-b-c \quad \mathbf{F}_{2} \cap d-\mathbf{F}_{2}-a-b-c$

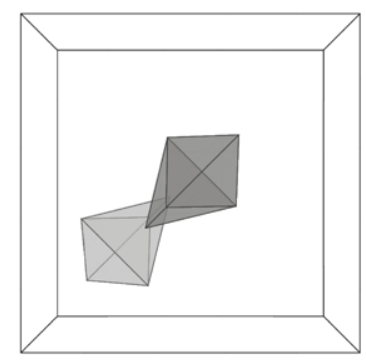

$+x+y-5 z=6$

Fig. A2 ${ }^{-}$The neighbors, intersection equations and separating planes of $\mathbf{F}_{2}$ (negative layers) 

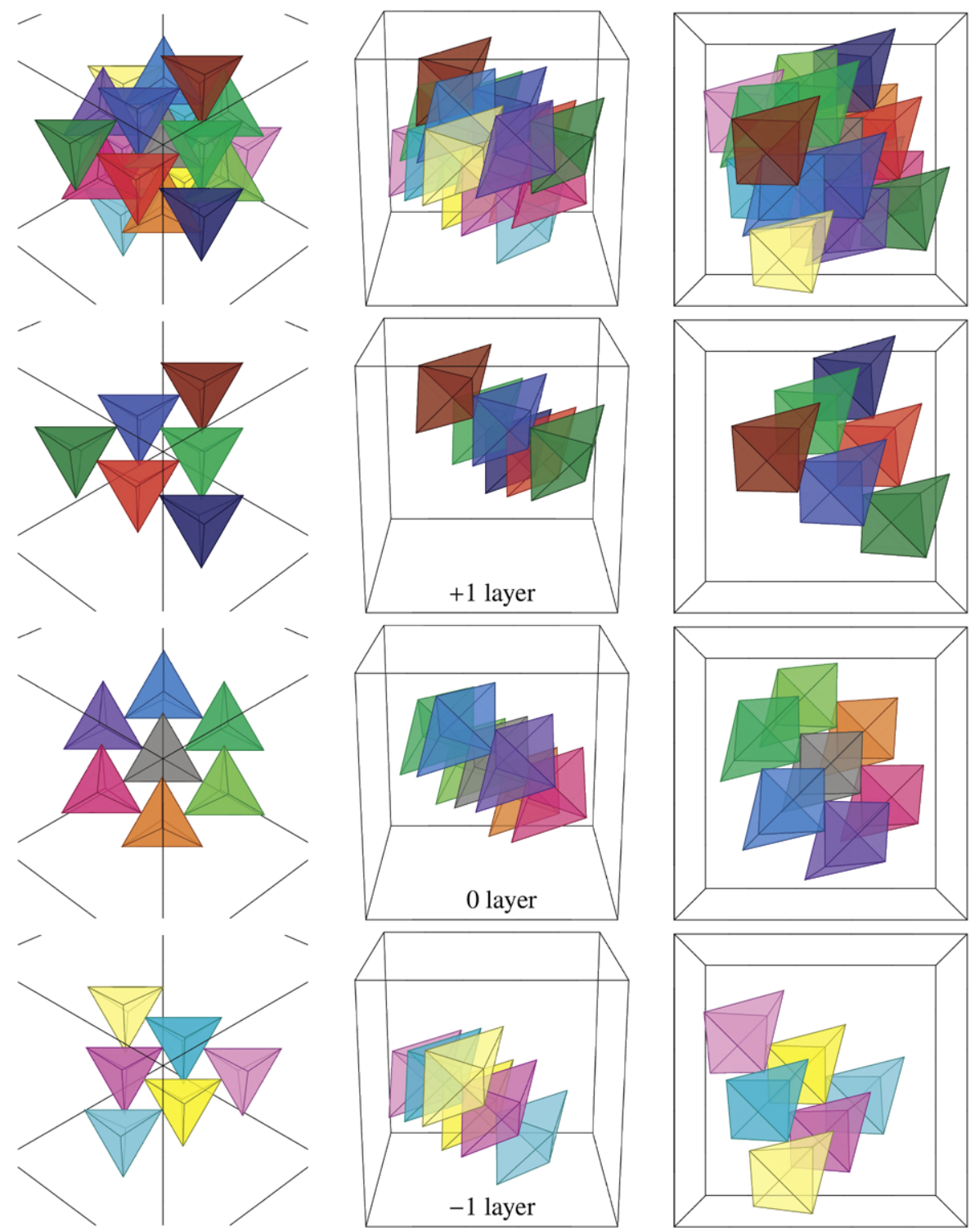

Fig. $\mathbf{A 3}_{\text {opt }}^{+}$Layers of the optimal packing $\langle u, v, w\rangle=\left\langle+\frac{3}{160},+\frac{3}{64}, 0\right\rangle$ 

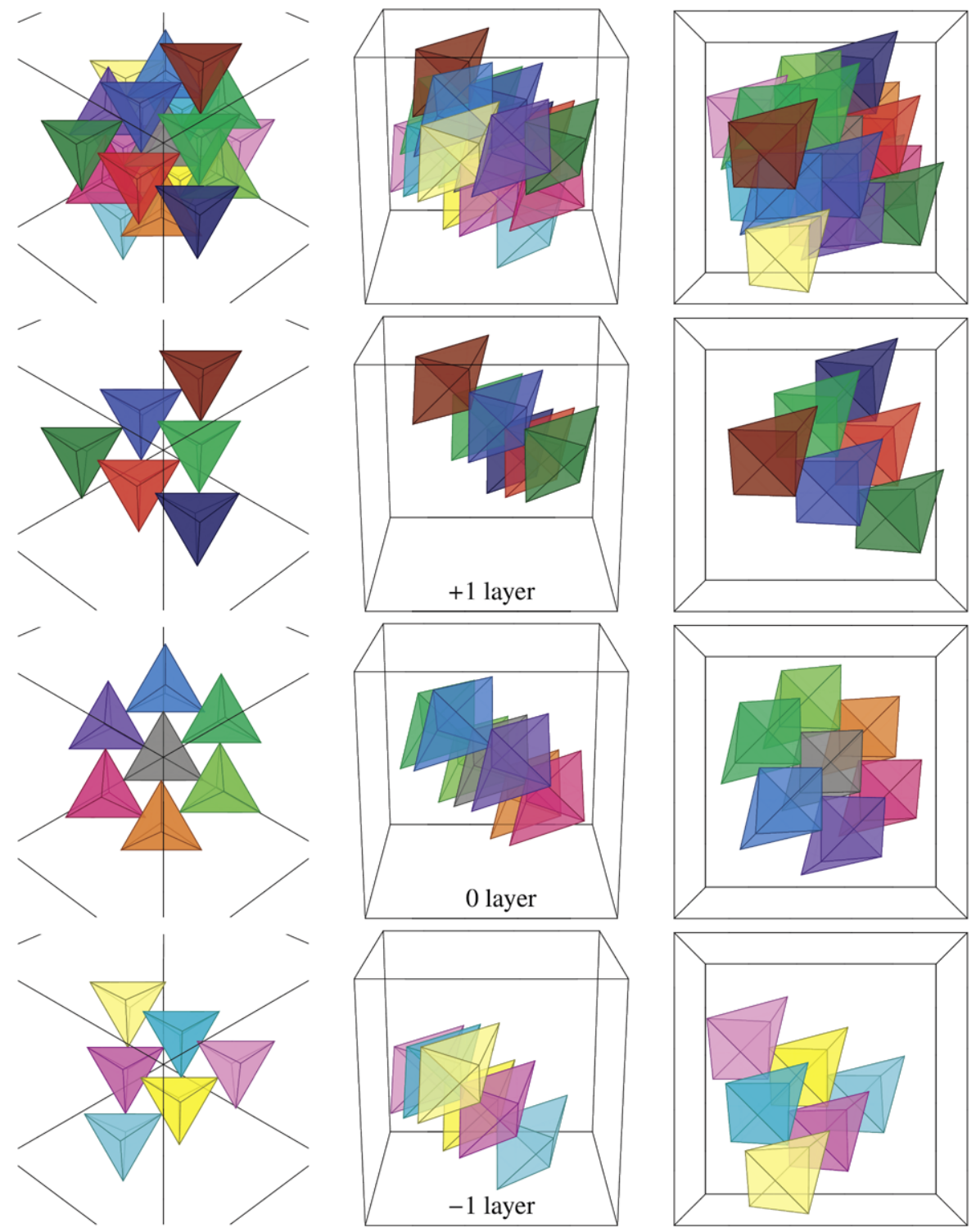

Fig. $\mathbf{A 3}_{\text {opt }}^{-}$Layers of the optimal packing $\langle u, v, w\rangle=\left\langle-\frac{3}{160},-\frac{3}{64}, 0\right\rangle$ 


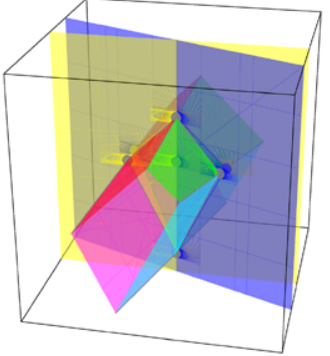

optimal plane

central plane

boundary plane

boundary plane

boundary plane

boundary plane

symmetric line

minimal line

minimal line

maximal line

maximal line

origin $\langle 0,0,0\rangle$

optimal point

optimal point

central point

central point

symmetric point

symmetric point $\partial V$

$\mathbf{H}_{a-b}$

$\mathbf{H}_{b+c}$

$\mathbf{H}_{c+a}$

$\mathbf{H}_{b-c}$

$\mathbf{H}_{c-a}$

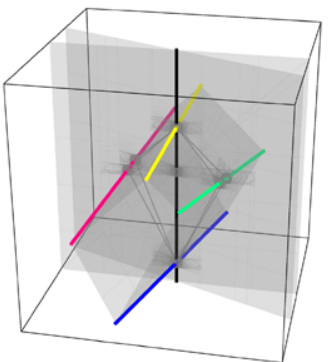

$\mathbf{H}_{a-b} \wedge \partial V$

$\mathbf{H}_{b-c} \wedge \mathbf{H}_{c-a}$

$\mathbf{H}_{b+c} \wedge \mathbf{H}_{c+a}$

$\mathbf{H}_{b+c} \wedge \mathbf{H}_{c-a}$

$\mathbf{H}_{b-c} \wedge \mathbf{H}_{c+a}$

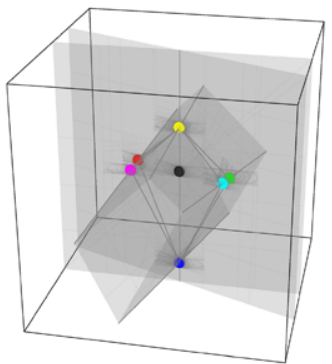

$\mathbf{H}_{b+c} \wedge \mathbf{H}_{c-a} \wedge \partial V$

$\mathbf{H}_{b-c} \wedge \mathbf{H}_{c+a} \wedge \partial V$

$\mathbf{H}_{b+c} \wedge \mathbf{H}_{c-a} \wedge \mathbf{H}_{a-b}$

$\mathbf{H}_{b-c} \wedge \mathbf{H}_{c+a} \wedge \mathbf{H}_{a-b}$

$\mathbf{H}_{b-c} \wedge \mathbf{H}_{c-a} \wedge \mathbf{H}_{a-b} \wedge \partial V$

$\mathbf{H}_{b+c} \wedge \mathbf{H}_{c+a} \wedge \mathbf{H}_{a-b} \wedge \partial V$

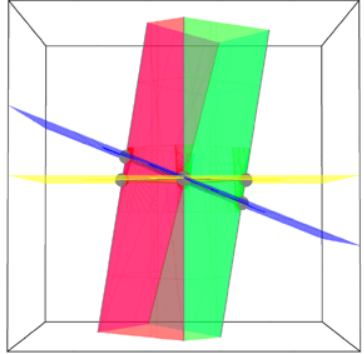

$5 u=2 v$

$u=0$

$+\frac{1}{2} u+2 v-w=\frac{33}{320}$

$-\frac{1}{2} u-2 v-w=\frac{33}{320}$

$-v+w=\frac{3}{64}$

$+v+w=\frac{3}{64}$

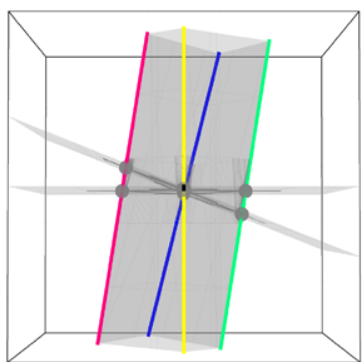

$\langle 0,0,0\rangle+w\langle 0,0,1\rangle$

$\left\langle 0,0,+\frac{3}{64}\right\rangle+u\langle 1,0,0\rangle$

$\left\langle 0,0,-\frac{33}{320}\right\rangle+u\left\langle 1,-\frac{1}{4}, 0\right\rangle$

$\left\langle+\frac{3}{160},+\frac{3}{64}, 0\right\rangle+u\left\langle 1,-\frac{1}{6},+\frac{1}{6}\right\rangle$

$\left\langle-\frac{3}{160},-\frac{3}{64}, 0\right\rangle+u\left\langle 1,-\frac{1}{6},-\frac{1}{6}\right\rangle$

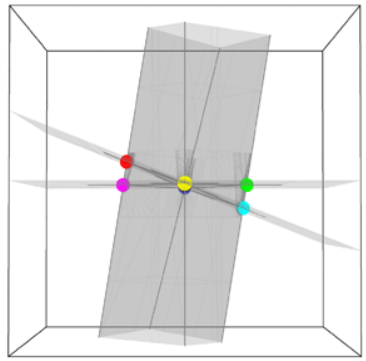

$\left\langle+\frac{3}{160},+\frac{3}{64}, 0\right\rangle$

$\left\langle-\frac{3}{160},-\frac{3}{64}, 0\right\rangle$

$\left\langle 0,+\frac{1}{20},-\frac{1}{320}\right\rangle$

$\left\langle 0,-\frac{1}{20},-\frac{1}{320}\right\rangle$

$\left\langle 0,0,+\frac{3}{64}\right\rangle$

$\left\langle 0,0,-\frac{33}{320}\right\rangle$

Fig. A4 Special planes, lines and points of the parameter space $\langle u, v, w\rangle$ 
$V=\frac{9}{25}\left(117+60 u^{2}-80 u v-80 v^{2}\right)$ $\phi=100 /\left(117+60 u^{2}-80 u v-80 v^{2}\right)$
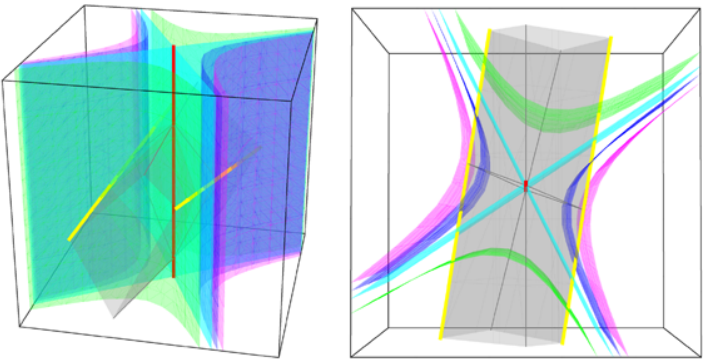

$V=\frac{42093}{1000}$

$\phi=\frac{4000}{4671}$

maximal lines

tangent to contour

optimal plane

contains tangent points

optimal points

$=$ tangent points

$\frac{1053}{25}>V>\frac{42093}{1000}$
$\frac{100}{117}<\phi<\frac{4000}{4671}$
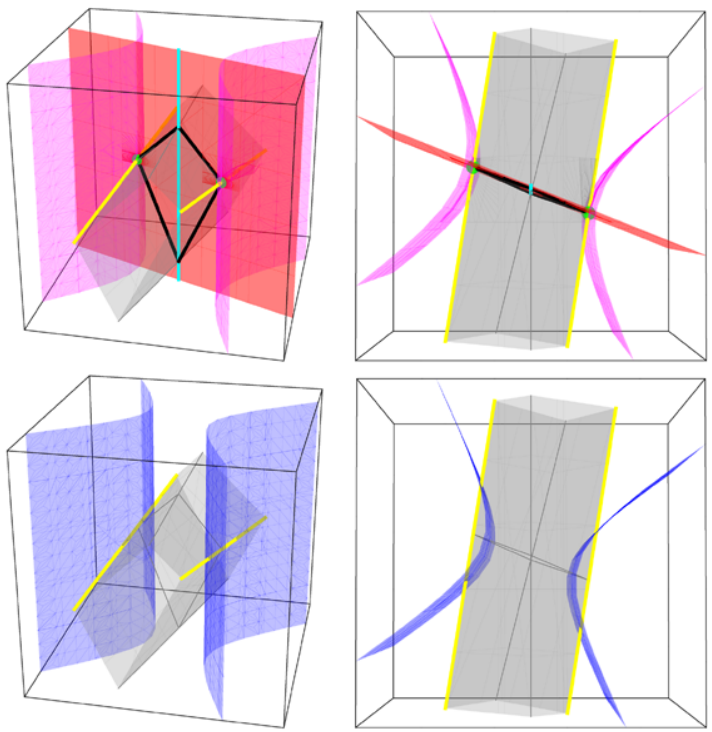

$V=\frac{1053}{25}$

$\phi=\frac{100}{117}$

asymptotes of contour

$=$ contour

symmetric line

$=$ central axis of contour symmetric points

@ central axis of contour

$V>\frac{1053}{25}$
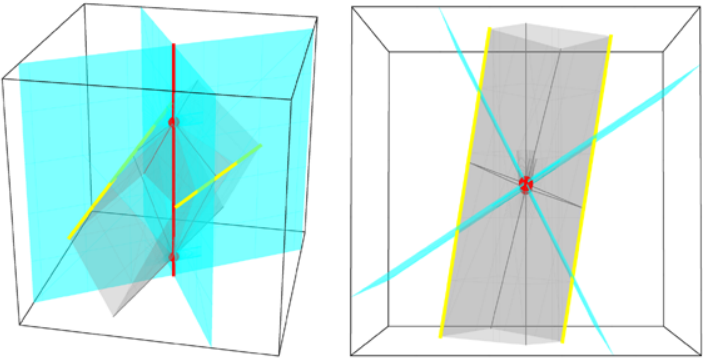

$\phi<\frac{100}{117}$
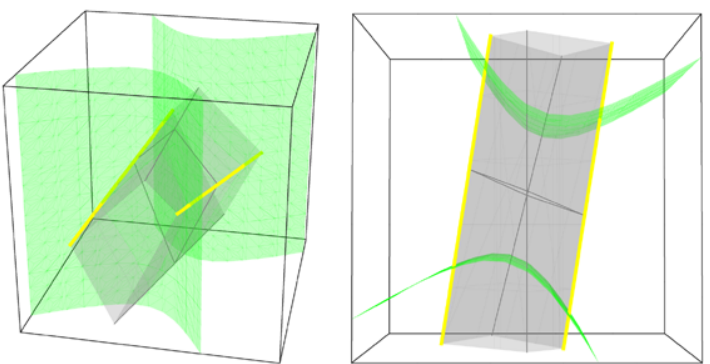

Fig. A5 Intersections of the parameter space $\langle u, v, w\rangle$ with contours of lattice volume $V$ 

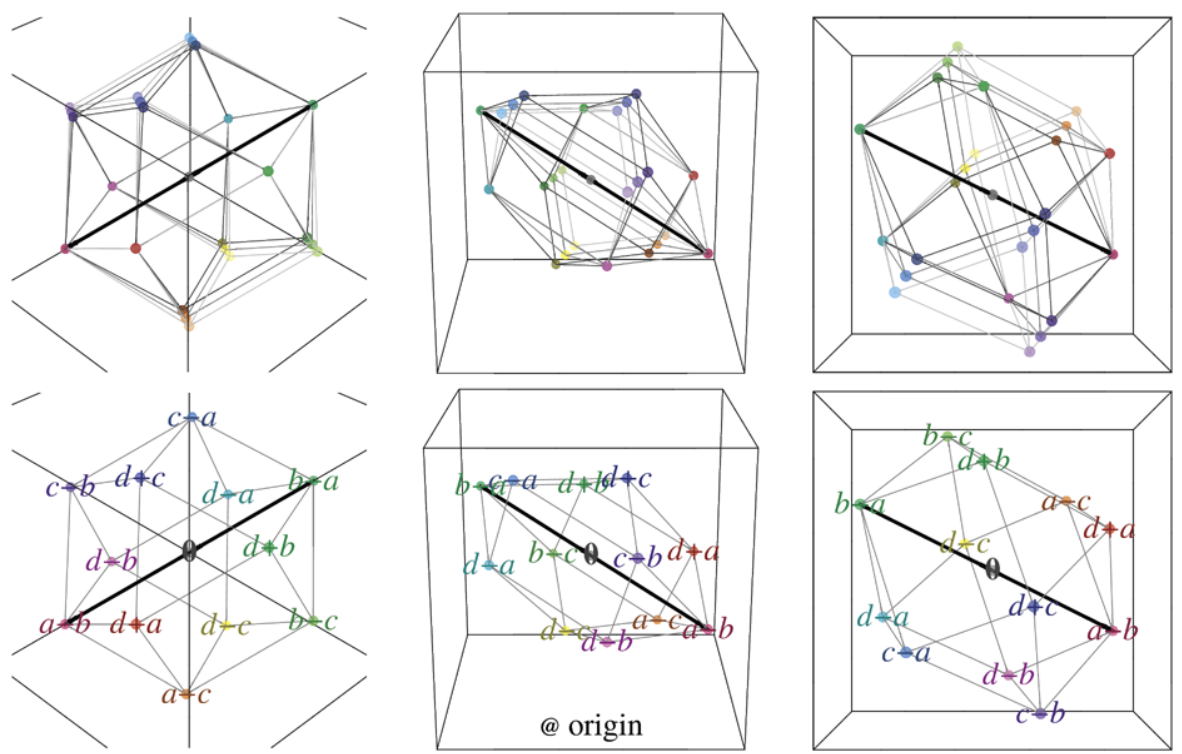

Fig. $\mathbf{A 6}_{\text {sym }}$ The axis (along $p=\langle+2,-1,-1\rangle$ ) of the symmetry $T$, and packings parametrized along the symmetric line $\langle 0,0,0\rangle+w\langle 0,0,1\rangle$
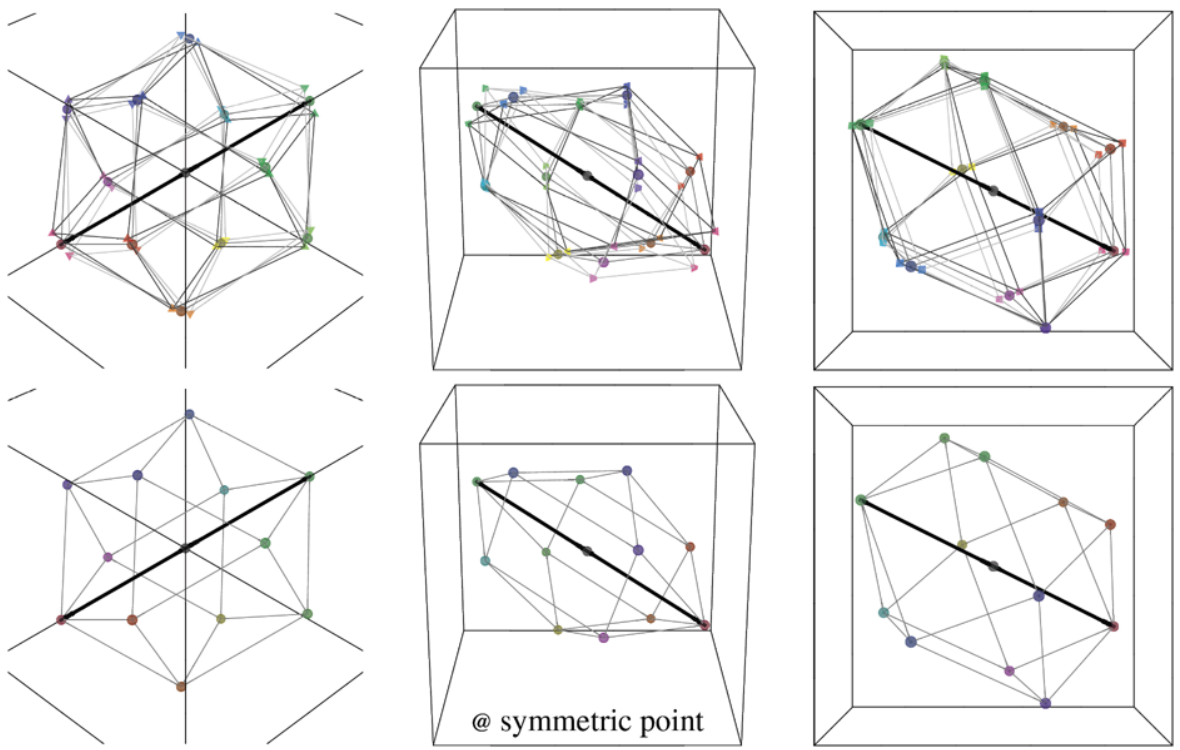

Fig. $\mathbf{A 6}_{\text {min }}^{+}$The axis (along $p=\langle+2,-1,-1\rangle$ ) of the symmetry $T$, and packings parametrized along the minimal line $\left\langle 0,0,+\frac{3}{64}\right\rangle+u\langle 1,0,0\rangle$ 

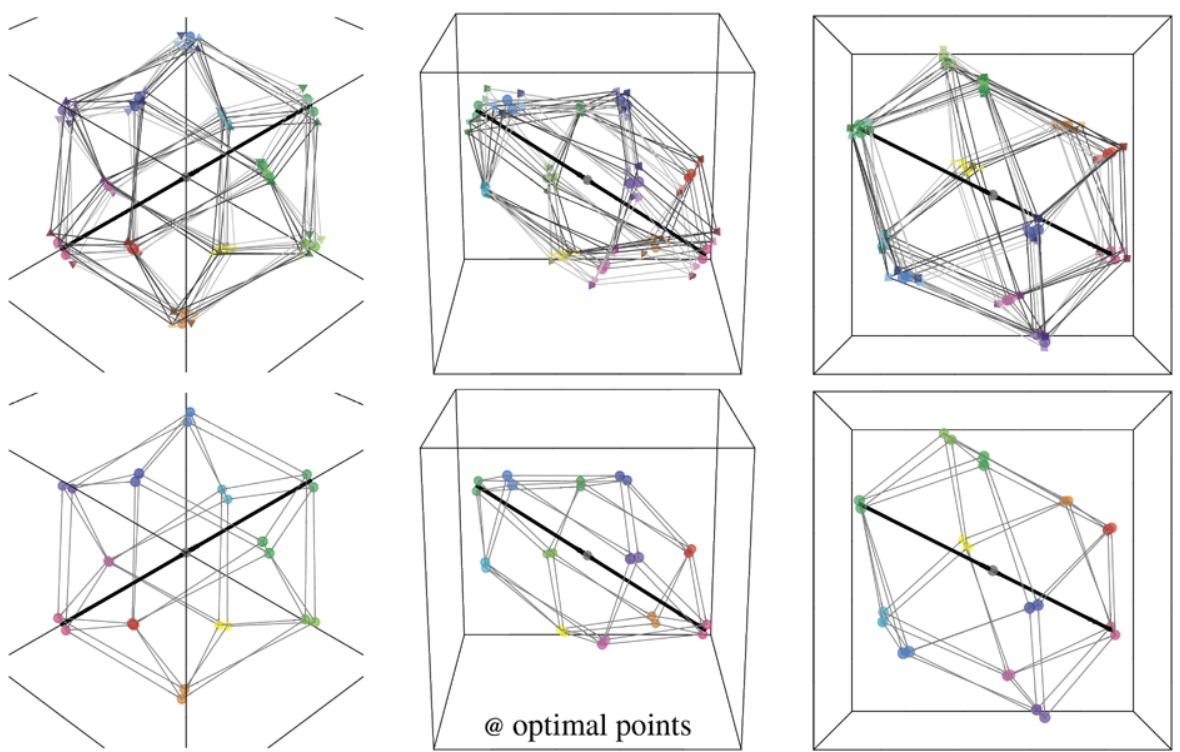

Fig. A6 $6_{\max }^{ \pm}$The axis (along $p=\langle+2,-1,-1\rangle$ ) of the symmetry $T$, and packings parametrized along the maximal lines $\left\langle+\frac{3}{160},+\frac{3}{64}, 0\right\rangle+u\left\langle 1,-\frac{1}{6},+\frac{1}{6}\right\rangle$ and $\left\langle-\frac{3}{160},-\frac{3}{64}, 0\right\rangle+u\left\langle 1,-\frac{1}{6},-\frac{1}{6}\right\rangle$ (in opposite directions)
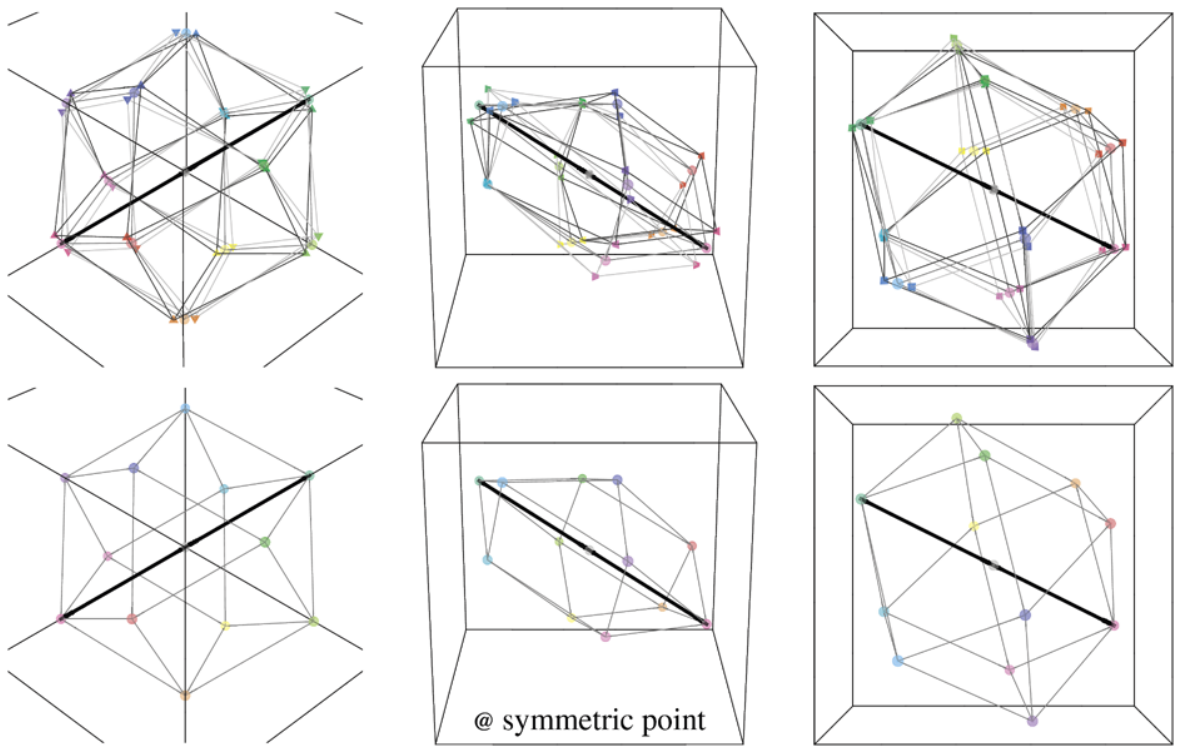

Fig. $\mathbf{A 6}_{\text {min }}^{-}$The axis (along $p=\langle+2,-1,-1\rangle$ ) of the symmetry $T$, and packings parametrized along the minimal line $\left\langle 0,0,-\frac{33}{320}\right\rangle+u\left\langle 1,-\frac{1}{4}, 0\right\rangle$ 


$$
\begin{aligned}
& T=\frac{1}{3} \begin{array}{lll}
+1 & -2 & -2 \\
-2 & -2 & +1 \\
-2 & +1 & -2
\end{array} \\
& \begin{array}{lll}
-2 & +1 & -2
\end{array} \\
& T: d, a, b, c \rightarrow d,-b,-a,-c \\
& \text { fixed line } p=\langle+2,-1,-1\rangle
\end{aligned}
$$

any 2 packings related by

$\langle\bar{u}, \bar{v}, \bar{w}\rangle=\langle-u,-v,+w\rangle$

fixed line $\langle 0,0,0\rangle+w\langle 0,0,1\rangle$

fixed planes $w=*$

has symmetry $T$

symmetric line

sym to self

origin

$$
\text { @ } w=0
$$

sym to self

minimal point

@ $w=+\frac{3}{64}$

sym to self

minimal point

@ $w=-\frac{33}{320}$

sym to self

minimal line

$$
\text { @ } w=+\frac{3}{64}
$$

sym to opposite point

@ same minimal line

minimal line

@ $w=-\frac{33}{320}$

sym to opposite point

@ same minimal line

\section{2 maximal lines}

skew lines, no single plane spans family of planes sym to opposite point

@ opposite maximal line

2 optimal points

@ $w=0$

sym to each other

2 central points

$$
\begin{aligned}
& @ w=-\frac{1}{320} \\
& \text { sym to each other }
\end{aligned}
$$
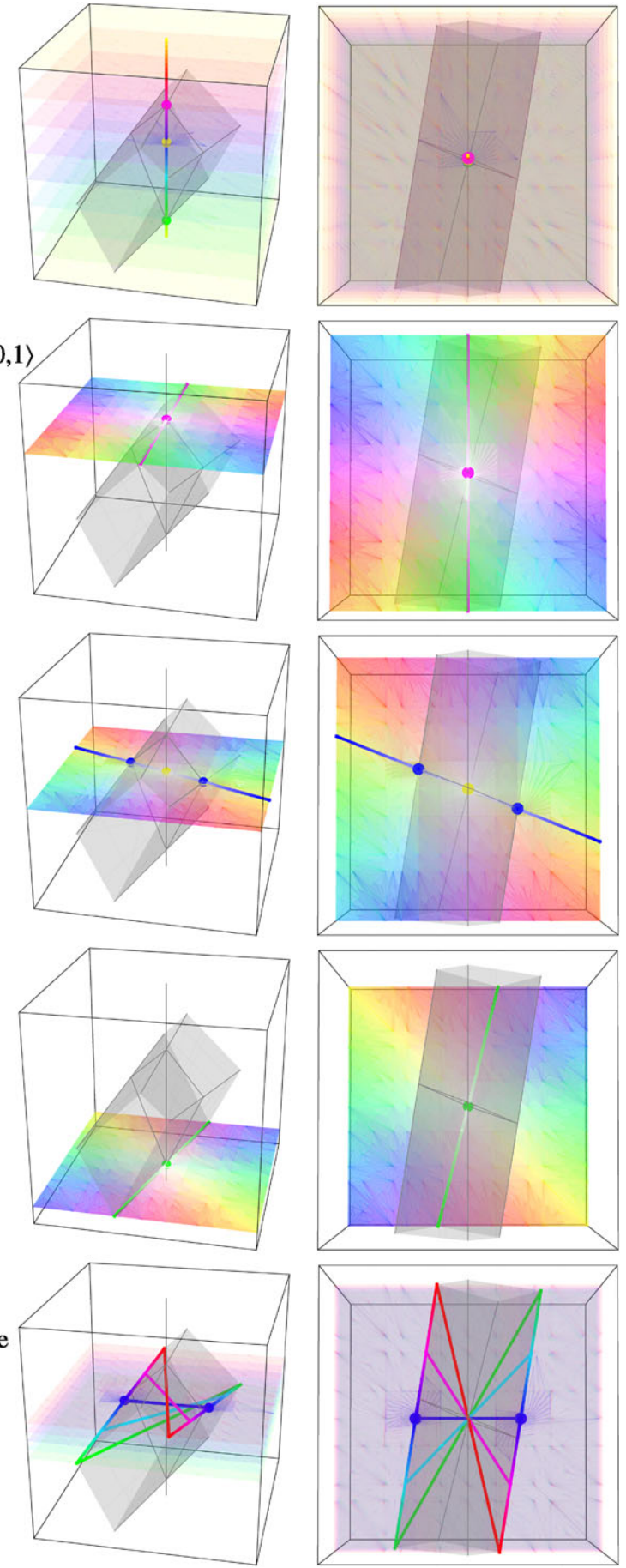

Fig. A7 Packing having symmetry $T$, or packing pairs related by the symmetry $T$ 